NBER WORKING PAPER SERIES

\title{
BAYESIAN ADAPTIVE CLINICAL TRIALS FOR ANTI INFECTIVE THERAPEUTICS DURING EPIDEMIC OUTBREAKS
}

\author{
Shomesh Chaudhuri \\ Andrew W. Lo \\ Danying Xiao \\ Qingyang Xu \\ Working Paper 27175 \\ http://www.nber.org/papers/w27175 \\ NATIONAL BUREAU OF ECONOMIC RESEARCH \\ 1050 Massachusetts Avenue \\ Cambridge, MA 02138 \\ May 2020
}

We thank Murray Sheldon, Chi Heem Wong, the editor, associate editor, and several reviewers for many helpful comments and suggestions, and Jayna Cummings, Steven Finch, and Roseann Staplins for editorial assistance. Research support from the MIT Laboratory for Financial Engineering is gratefully acknowledged. The views and opinions expressed in this article are those of the authors only, and do not represent the views, policies, and opinions any institution or agency, any of their affiliates or employees, or any of the individuals acknowledged above. Funding support from the MIT Laboratory for Financial Engineering is gratefully acknowledged, but no direct funding was received for this study and no funding bodies had any role in study design, data collection and analysis, decision to publish, or preparation of this manuscript. The authors were personally salaried by their institutions during the period of writing (though no specific salary was set aside or given for the writing of this manuscript). The views expressed herein are those of the authors and do not necessarily reflect the views of the National Bureau of Economic Research.

At least one co-author has disclosed a financial relationship of potential relevance for this research. Further information is available online at http://www.nber.org/papers/w27175.ack

NBER working papers are circulated for discussion and comment purposes. They have not been peer-reviewed or been subject to the review by the NBER Board of Directors that accompanies official NBER publications.

(C) 2020 by Shomesh Chaudhuri, Andrew W. Lo, Danying Xiao, and Qingyang Xu. All rights reserved. Short sections of text, not to exceed two paragraphs, may be quoted without explicit permission provided that full credit, including $\odot$ notice, is given to the source. 
Bayesian Adaptive Clinical Trials for AntiInfective Therapeutics during Epidemic Outbreaks Shomesh Chaudhuri, Andrew W. Lo, Danying Xiao, and Qingyang Xu

NBER Working Paper No. 27175

May 2020

JEL No. C11,C12,C44,C54,C9,C93,H12,H51,I1,I11,I12,I15,I18

\begin{abstract}
$\underline{\text { ABSTRACT }}$
In the midst of epidemics such as COVID-19, therapeutic candidates are unlikely to be able to complete the usual multiyear clinical trial and regulatory approval process within the course of an outbreak. We apply a Bayesian adaptive patient-centered model-which minimizes the expected harm of false positives and false negatives - to optimize the clinical trial development path during such outbreaks. When the epidemic is more infectious and fatal, the Bayesian-optimal sample size in the clinical trial is lower and the optimal statistical significance level is higher. For COVID-19 (assuming a static $R_{0}-2$ and initial infection percentage of $0.1 \%$ ), the optimal significance level is $7.1 \%$ for a clinical trial of a nonvaccine anti-infective therapeutic and $13.6 \%$ for that of a vaccine. For a dynamic $R_{0}$ decreasing from 3 to 1.5, the corresponding values are $14.4 \%$ and $26.4 \%$, respectively. Our results illustrate the importance of adapting the clinical trial design and the regulatory approval process to the specific parameters and stage of the epidemic.
\end{abstract}

Shomesh Chaudhuri

QLS Advisors

245 Main Street

Cambridge, MA 02142

shomesh@qlsadvisors.com

Andrew W. Lo

MIT Sloan School of Management

100 Main Street, E62-618

Cambridge, MA 02142

and NBER

alo-admin@mit.edu
Danying Xiao

Laboratory for Financial Engineering

100 Main Street

Cambridge, MA 02142

d6xiao@mit.edu

Qingyang Xu

Laboratory for Financial Engineering

100 Main Street

Cambridge, MA 02142

qxu94@mit.edu 


\section{Table of Contents}

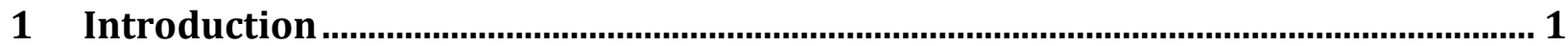

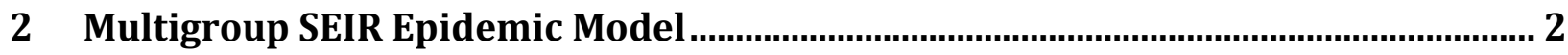

3 A Bayesian Patient-Centered Approval Process........................................................ 4

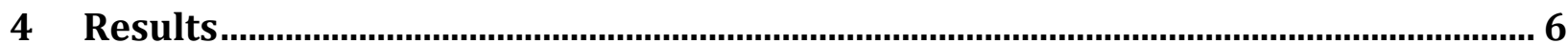

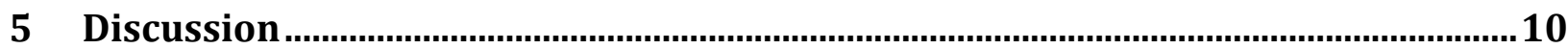

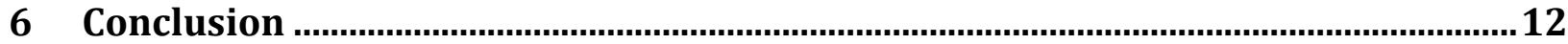

Author Disclosure Statements......................................................................................22

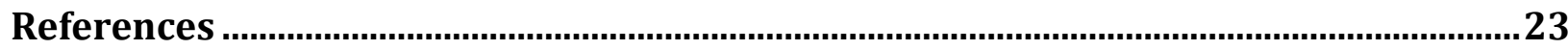

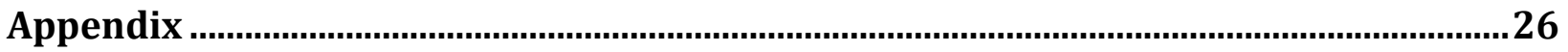




\section{Introduction}

With growing public concern over the outbreak of Coronavirus Disease 2019 (COVID-19), significant efforts have been undertaken by global biomedical stakeholders to develop effective diagnostics, vaccines, antiviral drugs, medical devices, and other therapeutics against this highly infectious and deadly pandemic. While in the past, the traditional randomized clinical trial (RCT) and regulatory approval process often took several years (U.S. Food \& Drug Administration, 2018)-longer than the typical duration of an epidemic outbreak (Pronker et al., 2013) - recently the FDA has responded with actions such as the Breakthrough Devices Program, Emergency Use Authorization (EUA) authority, and Immediately in Effect guidance documents to prevent novel diagnostics and therapeutics from lagging behind the urgent needs of the population. In this article, we propose adapting

yet another tool that the FDA has already been exploring for medical devices (Chaudhuri et al., 2018, 2019) to therapeutics for treating COVID-19 that are currently under development.

In recent years, Bayesian adaptive RCT protocols have been increasingly used to expedite the clinical trial process of potentially transformative therapies for diseases with high mortality rates (Berry, 2015). Currently, these protocols have mainly been applied within the oncology domain, such as I-SPY for breast cancer (Barker et al., 2009) and GBM AGILE for glioblastoma (Alexander et al., 2018). These studies use Bayesian inference algorithms to greatly reduce the number of patients needed to assess the therapeutic effects of a drug candidate, without lowering the statistical power of the final approval decision, as measured by Type I and II error rates. As a result, therapeutic candidates can progress more quickly through the regulatory process and reach patients faster and at lower costs.

For severe diseases with no curative treatments, such as pancreatic cancer, patients tend to tolerate a higher Type I error of accepting an ineffective therapy in exchange for a lower Type II error of rejecting an effective therapy as well as expedited approvals of potentially effective treatments. Based on this observation, a patient-centered Bayesian protocol was proposed (Isakov et al., 2019; Montazerhodjat et al., 2017) that incorporates patient values into clinical trial design and identifies the optimal balance between the possibilities of false positives (Type I error) and false negatives (Type II error). For more severe diseases, this protocol sets a tolerated Type I error rate much larger than the traditional 5\% threshold, which leads to higher rates of approvals and expedited approval decisions.

However, the original Bayesian adaptive RCT framework does not take into account patient risk preferences. To address this gap, Chaudhuri and Lo (2018) developed an adaptive version of the Bayesian patient-centered model that achieves an optimal balance between Type I and Type II error rates, significantly reducing the number of subjects needed in trials to achieve a statistically significant conclusion. A key feature of this model is the time evolution of the loss function of the Bayesian decision algorithm. This mechanism favors the

13 May 2020

(C) 2020 by Chaudhuri, Lo, Xiao, and Xu All Rights Reserved

Page 1 of 32 
expedited approval of diagnostic or therapeutic candidates that show early positive effects, since patients place a lower value on delayed approval of an effective diagnostic or therapy.

There is a natural but subtle analog to this dilemma in the case of therapeutics for an infectious disease during the course of an epidemic outbreak. Approving an effective therapeutic early will prevent future infections and deaths, while approving it later will save fewer people from infection. On the other hand, approving an ineffective therapeutic early will not prevent any future casualties. Worse still, it may prevent people from taking adequate precautions against infection, since they will falsely believe that they are safe from the disease after the advent of the ineffective therapy.

Moreover, the cost of Type I versus Type II error can differ from therapy to therapy. A novel vaccine that could trigger a significant immune response such as a cytokine storm has a much higher cost of a Type I error than a medical device such as an air filtration system designed to destroy virions through intense ultraviolet light. Therefore, the appropriate statistical threshold for approval should depend on the specific therapy, as well as the circumstances of the current burden of disease.

In this article, we apply the Bayesian adaptive protocol to anti-infective therapeutic development using a loss function that evolves over the course of an epidemic outbreak. We achieve an optimal balance between Type I and Type II errors for therapeutics that treat infectious diseases and identify the optimal time to reach the approval decision based on the accumulation of clinical evidence. Our results show that when the epidemic is more infectious, the necessary sample size of the RCT decreases, while the tolerable Type I error increases. This confirms our earlier intuition that potentially effective therapies that are known to be safe should receive expedited approval when an epidemic is spreading rapidly.

\section{Multigroup SEIR Epidemic Model}

The starting point for our analysis is the Susceptible-Exposure-Infective-Removed (SEIR) epidemic model, which has been applied to model the outbreak of COVID-19 in China in a number of recent studies (Wu et al., 2020; Yang et al., in press). The population of $N$ subjects is partitioned into four distinct groups: susceptible (S), exposed (E), infectious (I), and removed (R). The time evolution of the epidemic is specified by the following group of ordinary differential equations:

$$
\frac{d S}{d t}=-\beta S I, \quad \frac{d E}{d t}=\beta S I-a E, \quad \frac{d I}{d t}=a E-\gamma I, \quad \frac{d R}{d t}=\gamma I .
$$

13 May 2020

(c) 2020 by Chaudhuri, Lo, Xiao, and Xu

All Rights Reserved

Page 2 of 32 
Here we use the convention that $S(t), E(t), I(t)$, and $R(t)$ are the proportions of the susceptible, exposed, infectious and removed populations, respectively, satisfying the conservation constraint for all $t$ :

$$
S(t)+E(t)+I(t)+R(t)=100 \% .
$$

The parameters $\beta, a$, and $\gamma$ denote the average rates of infection, incubation, and recovery, respectively, and $\mu \in(0 \%, 100 \%)$ denotes the mortality rate of the epidemic. For example, if $\mu=5 \%$, we expect $5 \%$ of infected subjects will die from the disease. At time $t, \mu R(t) N$ subjects will have died, and $(1-\mu) R(t) N$ will have recovered.

A critical measure of the infectivity of an epidemic is its basic reproduction number, defined as $R_{0}=\beta / \gamma$ in the SEIR model. This is the expected number of secondary infections caused by each infected subject in a population with no public health measures (such as quarantine, social distancing, or vaccination).

A number of studies have used different statistical schemes to estimate $R_{0}$ for COVID-19 during its initial outbreak period in central China in January 2020. These estimated values of $R_{0}$ range from 2.2 ( $95 \% \mathrm{CI}, 1.4$ to 3.9) (Li et al., 2020) to 3.58 (95\% CI, 2.89 to 4.39) (Zhao et al., 2020). Given the large uncertainty in the value of $R_{0}$, we simulate therapeutic development under scenarios with constant $R_{0}$ values of 2 and 4.

In addition, to model the impact of governmental nonpharmaceutical interventions (NPIs) on containing the spread of the epidemic, we consider a dynamic transmission SEIR model where the infection rate, $\beta(t)$, monotonically decreases over time as a result of the NPIs. Specifically, we assume that $\beta(t)$ takes the sigmoid functional form:

$$
\beta(t)=\frac{\beta_{0}-\beta_{\infty}}{1+\exp \left(\frac{t-t_{2}}{\tau}\right)}+\beta_{\infty} .
$$

Here $\beta_{0}$ and $\beta_{\infty}$ denote the infection rates in the initial and final stages of the epidemic (with $\beta_{0}>\beta_{\infty}$ ), respectively, $t_{2}$ denotes the half-life of the decay in infection rate, and $\tau$ the length of the time window when this decay occurred. A larger difference between $\beta_{0}$ and $\beta_{\infty}\left(\beta_{0}-\right.$ $\beta_{\infty}$ ) corresponds to a more significant reduction of epidemic transmission, a smaller value of $t_{2}$ corresponds to a speedier decision to enforce the NPIs, and a smaller value of $\tau$ corresponds to more strict enforcement of the NPIs since $\beta(t)$ decays more rapidly. We calibrate the values $\beta_{0}=3$ and $\beta_{\infty}=1.5$ based on the estimates of the dynamic transmission rate of COVID-19 in Wuhan, China, from December 2019 to February 2020

13 May 2020

(C) 2020 by Chaudhuri, Lo, Xiao, and Xu All Rights Reserved

Page 3 of 32 
(Kucharski et al., 2020). We consider different values of $t_{2}$ and $\tau$ to reflect the variability in timing and stringency of NPIs enforced by governments around the globe. Under this dynamic transmission model, the basic reproduction number is given by $R_{0}(t)=\beta(t) / \gamma$, which monotonically decreases from $\beta_{0} / \gamma$ to a constant value $\beta_{\infty} / \gamma$ as $t$ increases.

To model the significant variability in mortality rates of COVID-19 for patients in different age groups, we extend this basic model to a multigroup SEIR model, where the population is partitioned into five age groups, (1) below 49, (2) 50 to 59, (3) 60 to 69, (4) 70 to 79, and (5) above 80 . We use $S_{i}, E_{i}, I_{i}$, and $R_{i}$ to denote the corresponding type in each group (and continue to use $S, E, I$, and $R$ for the total proportion of each type in all groups). The dynamics of the epidemic are specified by the modified ordinary differential equations:

$$
\frac{d S_{i}}{d t}=-\beta c_{i} S_{i} I, \quad \frac{d E_{i}}{d t}=\beta c_{i} S_{i} I-a E_{i}, \quad \frac{d I_{i}}{d t}=a E_{i}-\gamma I_{i}, \quad \frac{d R_{i}}{d t}=\gamma I_{i} .
$$

Here $c_{i}$ denotes the contact rate of the susceptible subjects in the $i^{\text {th }}$ age group with the total infected population, $I$, of all groups. This contact rate is measured relative to group 1 , which we normalize to $c_{1}=1$. In the case of COVID-19, although the mortality rate is much higher for senior populations (Onder et al., 2020), elderly people also tend to have less frequent contact with the infected population outside the household (Walker et al., 2020).

We solve the differential equations in the multigroup SEIR model using the ODE45 solver in MATLAB 2019a with initial conditions for each age group:

$$
\left[S_{i}(0), E_{i}(0), I_{i}(0), R_{i}(0)\right]=\left[1-\left(1+r_{e}\right) I_{0}, r_{e} I_{0}, I_{0}, 0\right] \times P_{i} .
$$

The parameter $I_{0}$ denotes the proportion of the initially infected population, $r_{e}$ is the ratio of initially exposed and infected subjects, and $P_{i}$ is the percentage of the $i^{\text {th }}$ age group in the population. The assumed demographic, contact rate, and mortality rate values are summarized in Table 1.

\section{A Bayesian Patient-Centered Approval Process}

Similar to Chaudhuri and Lo (2018), we develop a Bayesian patient-centered decision model for RCT approval that minimizes the expected loss (or harm) incurred on the patients by optimally balancing the losses of Type I and Type II errors. Here the loss does not refer to financial costs afforded by the patients, but rather the loss in patient value (i.e., how much patients weigh the relative harms of infection and death). We assign the losses per patient of 
being susceptible, infected, and deceased. Since Bayesian decision thresholds are invariant under the rescaling of the losses, we normalize by setting the loss per patient infection to 1 $\left(L_{I}=1\right)$. We then assign the loss per patient death relative to $L_{I}$ as $L_{D}$, and the loss due to susceptibility to the disease as $L_{S}$. The parameter values we assume, summarized in Table 2 , are meant to represent one reasonable valuation of the relative losses. However, in practice, patient value will differ from one patient group to another, especially given the large variability of the mortality rate of COVID-19 in different age groups (Onder et al., 2020). Here we report the main results of optimal sample size and statistical significance (Tables 3 and 4) assuming $L_{D}=100$. The results for $L_{D}=10$ are provided in the Appendix.

We simulate the multigroup SEIR model over a time period of $T$ weeks, where $T$ is the duration of the epidemic outbreak. Let $\kappa$ denote the weekly subject enrollment rate in each arm of the clinical trial. We assume that the value of $R_{0}$ is known (or well-estimated) at initial time $t=0$ and stays constant during the course of the outbreak. At time $t \in[0, T]$, the Bayesian loss, $C_{i j}(t)$, of choosing action $\widehat{H}=i$ under $H=j$ is defined as:

\begin{tabular}{|c|c|c|}
\hline & $\widehat{\boldsymbol{H}}=\mathbf{0}$ (do not approve) & $\widehat{\boldsymbol{H}}=\mathbf{1}$ (approve) \\
\hline $\boldsymbol{H}=\mathbf{0}$ (ineffective) & 0 & $(S(t)-S(T)) N L_{S}$ \\
\hline $\boldsymbol{H}=\mathbf{1}$ (effective) & $R(T) N\left(L_{I}+\mu L_{D}\right)$ & $C I(t) N L_{I}+\mu R(t) N L_{D}$ \\
& & \\
\hline
\end{tabular}

where we define the cumulative number of infected patients, $C I(t)$, until time $t$ :

$$
C I(t)=E(t)+I(t)+R(t) .
$$

By design, this loss function penalizes Type I errors early in the epidemic by the susceptible term, $(S(t)-S(T)) N L_{S}$. We subtract the base level $S(T)$ from $S(t)$ since the multigroup SEIR model predicts that $S(T) N$ subjects will not be infected by the epidemic. A Type I error at an earlier time will expose more currently susceptible populations to the epidemic, since members will falsely believe that they are safe from the disease after the advent of the ineffective therapeutic. On the other hand, the loss function also penalizes correct approval decisions made at later stages of an epidemic via the cumulative infected and death terms, 
$C I(t)$ and $\mu R(t)$. A correct but delayed approval decision for the therapeutic is less valuable since it will save fewer susceptible people from infection and death.

The Bayesian decision model considers the null hypothesis, $H=0$, that the anti-infective therapeutic (or vaccine) has no clinical effect, against the alternative hypothesis that it has positive clinical effect with signal-to-noise ratio $\rho$ (Chaudhuri \& Lo, 2018). We use $p_{0}$ and $p_{1}$ to denote the Bayesian prior probabilities of $H=0$ and $H=1$, respectively.

This patient-value model imposes higher losses for incorrect approvals at earlier stages and correct approvals at later stages of an epidemic. Under these constraints, the Bayesian decision algorithm yields the sample size and statistical significance threshold of the RCT that optimally balances Type I and Type II errors.

\section{Results}

We simulate an epidemic outbreak over a time period of $T$ weeks, where $T$ is the duration of the outbreak. For an epidemic with higher infectivity, its duration is shorter, which creates more pressure to reach a timely approval decision. To avoid numerical instability, we formally define $T$ as the time when the number of cumulative infected subjects first reaches $99.9 \%$ of total infections predicted by the SEIR model. We assume an age-specific mortality rate $\mu$ at the level of COVID-19 (Onder et al., 2020; World Health Organization, 2020), and incubation and recovery periods of 7 days each (Yang et al., in press). These estimated parameters can all be challenged to varying degrees, depending on the specific drugindication pair under consideration and the particular circumstances of the epidemic, but they are meant to be representative of a typical anti-infective therapeutic during the midst of a growing epidemic.

We also assume that it takes 7 days after injection to assess the efficacy of the therapeutic on each subject. We adopt the optimization scheme of Montazerhodjat et al. (2017) to find the optimal Type I and Type II error rates of the nonadaptive Bayesian RCT. To represent typical practice of the pharmaceutical industry, we optimize under the upper bound on the model's power, Power $r_{\max }=90 \%$ (Isakov et al., 2019). We then use these optimal error rates as our stopping criteria to simulate the sequential decision process of a Bayesian adaptive RCT via Monte Carlo simulation (Chaudhuri \& Lo, 2018). The simulation results are summarized in Tables 3 and 4.

We separate the results into two distinct types of therapeutics-nonvaccine anti-infectives (Table 3) and vaccines (Table 4) - because of the differences in their historical probabilities of success. Vaccine development programs have an estimated probability of success $p_{1}^{v a c}=$ $40 \%$ as of 2019Q4 (https://projectalpha.mit.edu), whereas the corresponding figure for nonvaccine anti-infectives is $p_{1}^{n v}=23 \%$ (Wong et al., 2020).

13 May 2020

(C) 2020 by Chaudhuri, Lo, Xiao, and Xu

All Rights Reserved

Page 6 of 32 


\subsection{Nonvaccine Anti-Infective Therapeutics}

\section{Static Transmission Rate}

We first analyze the case in which the infectivity $R_{0}$ remains constant over time (e.g., in the absence of effective NPIs). For the fixed-sample Bayesian RCT of a nonvaccine anti-infective therapeutic, as $R_{0}$ increases from 2 to 4 (rows 1 to 2 of Table 3), the optimal sample size of each experimental arm decreases from 242 to 158 and the optimal Type I error rate drastically increases from $7.1 \%$ to $17.3 \%$ (Figure 1), much higher than the traditional $5 \%$ threshold. As the epidemic spreads across the population more rapidly, the Bayesian RCT model exhibits greater pressure to expedite the approval process and a much higher tolerance of false positive outcomes.

For the Bayesian adaptive RCT, when the therapeutic is ineffective $(H=0)$, the average sample size required to reject the therapeutic is much smaller than that of the nonadaptive version (columns 7 and 8 of Table 3). Also, the required sample size decreases with the infectivity $R_{0}$ in both mean and quartiles, yet always achieves a Type I error rate $(\alpha)$ below that of the nonadaptive version (column 11). The adaptive Bayesian decision model is able to reject an ineffective therapeutic with a relatively small sample size and a bounded falsepositive rate.

On the other hand, when the therapeutic is effective ( $H=1)$, as $R_{0}$ increases from 2 to 4 , the average sample size required by the Bayesian adaptive RCT decreases from 148 to 98 (columns 9 and 10 of Table 3). The Bayesian adaptive model places more weight on approving an effective therapeutic earlier to prevent future infections when the epidemic is more infectious. Despite the smaller sample size, the model still retains an empirical power above $91.0 \%$ for all values of $R_{0}$ (column 12). The Bayesian adaptive model simultaneously expedites the approval of an effective therapeutic and retains a bounded false-negative rate. The results are illustrated in Figure 2.

Furthermore, as the proportion of the initially infected population $I_{0}$ decreases from $0.1 \%$ to $0.01 \%$ (rows 4 to 6 of Table 3), the optimal sample sizes for nonadaptive and adaptive RCTs both increase, while the optimal Type I error rates decrease. Beginning the clinical trials for a therapeutic during the earlier stages of an epidemic outbreak reduces the need to expedite the approval process in order to contain its future spread. Clinicians and researchers have more time to evaluate the efficacy of a therapeutic and record adverse effects by testing it on a larger number of subjects, which leads to a lower Type I error rate.

Finally, when the mortality rate $\mu$ increases from the level of COVID-19 (Onder et al., 2020; World Health Organization, 2020), to the level of SARS (World Health Organization, 2003), and further to the level MERS (World Health Organization, 2019), the optimal sample sizes for both nonadaptive and adaptive Bayesian models decrease and the optimal Type I error rates increase (rows 7 to 12 of Table 3). When the epidemic is more lethal, the Bayesian

13 May 2020

(C) 2020 by Chaudhuri, Lo, Xiao, and Xu All Rights Reserved

Page 7 of 32 
adaptive model requires fewer subjects in the RCT, since both Type I and Type II errors will lead to greater losses due to death by infection. The higher death tolls provide significantly more incentive in the Bayesian adaptive framework to approve the therapeutic in the hopes of saving more people from future infection and death.

One interesting feature of the Bayesian decision model is that the optimal Type I error rate is not a monotonic function of $R_{0}$, but rather has a minimum around $R_{0}=1.7$ for COVID-19, as shown in Figure 1. As $R_{0}$ decreases below 1.7, the optimal Type I error rate increases. The intuition for this result that we define the loss of Type I error as the excess risk of being susceptible to infection, $(S(t)-S(T)) N L_{S}$, where $S(T)$ is the fraction of the population that remains uninfected throughout the epidemic outbreak. When $R_{0}$ is small, $S(T)$ is close to $100 \%$ and the excess risk, $(S(t)-S(T)) N L_{S}$, is small compared to the benefit of preventing future deaths. Therefore, when the epidemic is not very infectious, the Bayesian decision model expedites the approval decision. This also confirms the intuition that smaller sample sizes are required in adaptive trials for diseases that affect a small fraction of the population. If we instead define the loss of Type I error as the absolute risk of being susceptible, $S(t) N L_{S}$, we find that the optimal Type I error indeed monotonically increases with $R_{0}$, as shown in Figure S3 in the Appendix.

\section{Dynamic Transmission Rate}

The results for the dynamic transmission model with $\beta_{0}=3, \beta_{\infty}=1.5, t_{2}=3$ weeks, and $\tau=$ 1 week are also summarized in Table 3. For COVID-19 (rows 3 and 6 in Table 3), we find that the Bayesian optimal sample size and Type I error rate of the dynamic transmission model lie in-between the corresponding values under scenarios $R_{0}=2$ and $R_{0}=4$. This suggests that timely and effective government interventions will protect more subjects from infection and allow more time for the RCT.

However, for the more fatal SARS and MERS (rows 9 and 12 in Table 3), the dynamic transmission model sets higher optimal Type I errors and smaller sample sizes than $R_{0}=4$. This is due to the U-shaped curve of optimal $\alpha$ vs. $R_{0}$, shown in Figure 1 . When the NPIs reduce $R_{0}(t)$ below a certain threshold, the optimal $\alpha$ starts to increase. For highly fatal epidemics, when the government adopts NPIs to protect most of the susceptible population from infection, the regulatory priority should be to expedite potentially effective treatments that can help current patients since the loss of Type I error is much lower than that of the Type II error.

In addition, we investigate the impact of the timing and stringency of NPIs enforced by the government with different values of $t_{2}$ and $\tau$. The results are summarized in Table 5 . We find that the optimal Type I error is larger for $t_{2}=3$ weeks than $t_{2}=6$ weeks. Therefore, if the government adopts well-enforced NPIs early on (such as the lockdown in Wuhan, China) to protect the susceptible population, this will reduce the loss associated with Type I error,

13 May 2020

(C) 2020 by Chaudhuri, Lo, Xiao, and Xu All Rights Reserved

Page 8 of 32 
leading to expedited approvals of potentially effective therapeutics. Furthermore, the sooner an effective therapeutic is approved, the sooner will NPIs be lifted.

\subsection{Vaccines}

We repeat the above analysis for an RCT of a vaccine using a prior probability of having an effective vaccine $p_{1}^{v a c}=40 \%$ as reported at https://projectalpha.mit.edu for 2019Q4. The simulation results are summarized in Table 4. Overall, we observe the same pattern in the optimal sample size and Type I error rates on infectivity, mortality, and proportion of initial infections. However, since $p_{1}$ is higher for vaccines, the Bayesian decision model requires fewer subjects, on average, in the RCT to ascertain the positive effects of the vaccine, compared to the case of anti-infective therapeutics in Table 3. We find that vaccines should receive even more expedited evaluation.

\subsection{Five-Factor Sensitivity Analysis}

To assess the robustness of our model's predictions against the assumed values of model parameters, we perform a five-factor sensitivity analysis for the static transmission rate model with $R_{0}=2$. The baseline and alternative parameter values are summarized in Table S1. The scatterplot of optimal Type I error $(\alpha)$ vs. sample size of Bayesian nonadaptive RCT model is shown in Figure 3 (related summary statistics are shown in Table S5 in the Appendix). We find that the scatterplot consists of several curves. To clearly identify the effect of any given parameter, we show the results for the most important parameters in separate scatterplots in Figures 4 to 6 (additional results are provided in the Appendix).

We find that the different curves in Figure 3 result from different values of $\rho$, the signal-tonoise ratio (SNR) of treatment effect (Chaudhuri \& Lo, 2018), as shown in Figure 4 . For a given significance level $\alpha$, a smaller value of $\rho$ leads to larger optimal sample size. If the efficacy of the anti-infective therapeutic is insignificant (small $\rho$ ), the distributions of $z$-score under the null hypothesis, $H=0$ (no effect), and alternative hypothesis, $H=1$ (positive effect with SNR $\rho$ ), are difficult to distinguish statistically. Hence, a larger sample size is needed to evaluate the efficacy at the given significance level, $\alpha$.

In addition, with a fixed SNR, the magnitudes of $\alpha$ and sample size are mainly determined by $p_{0}^{n v}$, the Bayesian prior probability of having an ineffective anti-infective therapeutic. A larger value of $p_{0}^{n v}$ leads to a smaller $\alpha$ and a larger sample size (Figure 5). When past drug development outcomes in the anti-infective domain strongly suggest that the current antiinfective therapeutic is unlikely to be effective (large $p_{0}^{n v}$ ), the Bayesian framework requires many more observations to shift the posterior distribution in order to prove its efficacy. For notational convenience, we denote $p_{0}^{n v}$ by $p_{0}$ in Figure 5.

A similar but less significant effect on the magnitudes of $\alpha$ and sample size is generated by $\kappa$, the weekly subject enrollment in each arm of the RCT. A larger value of $\kappa$ leads to a smaller

13 May 2020

(C) 2020 by Chaudhuri, Lo, Xiao, and Xu All Rights Reserved

Page 9 of 32 
$\alpha$ and a larger sample size (Figure 6). When the RCT enrolls patients at a faster rate, clinical researchers may evaluate the efficacy of the treatment based on more observations earlier on during the epidemic outbreak. Hence, the approval decision may be reached with lower false positive error.

In Figures S1 and S2 of the Appendix, we show that the five-factor sensitivity analysis reveals no significant dependence of $\alpha$ and sample size on $\Delta t$, the time needed to assess the treatment efficacy, as well as $a$, the incubation period of the disease. However, the analysis does show that extreme values of the Bayesian optimal Type I error rate are generated by large values of $p_{0}^{n v}$ and small values of $\rho$. These regions of parameter space can be avoided if the anti-infective therapeutic under investigation has promising preclinical evidence to support its efficacy (reducing $p_{0}^{n v}$ ) and the RCT is designed to verify reasonably significant treatment effects over the control arm (increasing $\rho$ ).

To provide readers with greater transparency and intuition for our Bayesian decision model, we provide the source code at https://projectalpha.mit.edu/resources, allowing users to input their own parameter values of interest to see how the results change. We also encourage users to adapt our code to their own contexts, as well as to experiment with alternate epidemiological models of infection and loss functions.

\section{Discussion}

A natural consequence of using a patient-centered framework for determining the approval threshold is, of course, more false positives-and the potential for a greater number of patients with adverse side-effects - in cases where the burden of disease is high. These false positives can be addressed through more vigilant postapproval surveillance by regulatory agencies and greater requirements for drug and device companies to provide such patientlevel data to the regulator following approval. Failure to provide such data or evidence of an ineffective therapy can be grounds for revoking the approval.

However, past experience shows that withdrawing an approved drug can be challenging and disruptive for several reasons (Onakpoya et al., 2019). Therefore, implementing the patientcentered approach may require creating a new category of temporary approvals for crisis situations involving urgent needs at national or international levels, similar to the FDA's EUA program. Such a program might involve provisional approval of a candidate therapy consisting of a one- or two-year license-depending on the nature of the drug-indication pair-to market the therapy to a prespecified patient population, no off-label use of the therapy, and regular monitoring and data reporting to the regulator by the manufacturer and/or patients' physicians during the licensing period (Lo, 2017). At the end of this trial period, one of two outcomes would occur, depending on the accumulated data during this period: (a) the 'urgent needs' license expires; or (b) the license converts to the traditional 
regulatory license. Of course, at any point during the trial period, the regulator can terminate the license if the data show that the therapeutic is ineffective and/or unsafe.

While such a process may impose greater burdens on patients, manufacturers, and regulators, it may still be worthwhile if it brings faster or greater relief to patients facing mortal illnesses and extreme suffering. In this respect, an urgent-needs program may be viewed as a middle ground between a standard clinical trial and an approval, similar in spirit to the adaptive designs of sophisticated clinical trials with master protocols such as I-SPY 2, LUNG-MAP, and GBM-AGILE, in which patient care and clinical investigations are simultaneously accomplished. Also, because the Centers for Medicare and Medicaid Services (CMS) has demonstrated a willingness to cover the cost of certain therapeutics for which evidence is still being generated (see, for example, CMS's "coverage with evidence" programs listed at https://go.cms.gov/2v6ZxWm), additional economic incentives may be available to support such temporary licenses.

Finally, we note that the age-group specification in our SEIR model mainly focuses on older populations, whose mortality risks with COVID-19 are much higher than younger populations (Onder et al., 2020). More refined age-group specifications are needed to differentiate the transmission rates of COVID-19 among children, teenagers, and young adults, as well as to reflect the different societal benefits each age group will receive from the approval of an effective anti-infective therapeutic or vaccine. 


\section{Conclusion}

We apply the Bayesian adaptive patient-centered model of Chaudhuri and Lo (2018) to clinical trials for therapeutics that treat infectious diseases during an epidemic outbreak. Using a simple epidemiological model, we find that the optimal sample size in the clinical trial decreases with the infectivity of the epidemic, measured by the basic reproduction number $R_{0}$. At the same time, the optimal Type I error rate increases with $R_{0}$. Lower levels of initial infection increase the number of subjects required to verify the therapeutic efficacy of the therapeutic under investigation, while higher levels of mortality increase the optimal sample size. The results confirm our intuition that clinical trials should be expedited and a higher false positive rate should be tolerated when the epidemic spreads more rapidly through the population, has a higher mortality rate, and has already infected a sizable portion of the population at the beginning of the RCT.

To provide transparency for how a patient-centered approach differs from the traditional statistical framework in the anti-infectives context, we use a relatively simple mathematical model of epidemic disease dynamics to estimate the societal loss in an outbreak. More sophisticated epidemiological models can easily be incorporated into our framework at the cost of computational tractability and transparency (see https://projectalpha.mit.edu/resources for details).

One interesting trade-off to be explored is the difference between a COVID-19 vaccine and an antiviral treatment that can cure an infected patient. While prevention through vaccination is the ultimate goal, a successful treatment for the disease using repurposed drugs that have already been approved for other indications (and whose safety profile has already been established) may be even more valuable, especially if they can be deployed in the nearer term and reduce the growing fear and panic among the general population. In such cases, the approval threshold should clearly reflect these cost-benefit differences.

Of course, in practice, regulators consider many factors beyond $p$ values in making their decisions. However, that process is opaque even to industry insiders, and the role of patient preferences is unclear. The proposed patient-centered approach provides a systematic, objective, adaptive, and repeatable framework for explicitly incorporating patient preferences and burden-of-disease data in the therapeutic approval process. This framework also fulfills two mandates for the FDA, one from the fifth authorization of the Prescription Drug User Fee Act (PDUFA) for an enhanced quantitative approach to the benefit-risk assessment of new drugs (U.S. FDA, 2013), and the other from Section 3002 of the 21st Century Cures Act of 2016 requiring the FDA to develop guidelines for patientfocused drug development, which includes collecting patient preference and experience data and explicitly incorporating this information in the drug approval process. 
We hope this work will shed further insight into improving the current clinical trial process for infectious disease therapeutics and contribute to the timely development of effective treatments and vaccines for COVID-19 in particular. 
Table 1. Demographic (U.S. Census Bureau, 2018), Relative Contact Rate (Walker et al., 2020), and Mortality (Onder et al., 2020; World Health Organization 2003, 2019, 2020) Profile of Various Age Groups for COVID-19, SARS, and MERS

\begin{tabular}{|c|c|c|c|c|}
\hline Age Group & $\begin{array}{c}\text { Percentage of } \\
\text { U.S. Population } \\
P_{i}\end{array}$ & $\begin{array}{c}\text { Contact Rate } \\
c_{i}\end{array}$ & Disease & $\begin{array}{c}\text { Mortality } \\
\mu_{i}\end{array}$ \\
\hline \multirow{3}{*}{ Below 49} & \multirow{3}{*}{$64 \%$} & \multirow{3}{*}{1.00} & COVID-19 & $0.3 \%$ \\
\hline & & & SARS & $3 \%$ \\
\hline & & & MERS & $15 \%$ \\
\hline \multirow{3}{*}{$50-59$} & \multirow{3}{*}{$13 \%$} & \multirow{3}{*}{0.83} & COVID-19 & $1.3 \%$ \\
\hline & & & SARS & $10 \%$ \\
\hline & & & MERS & $30 \%$ \\
\hline \multirow{3}{*}{$60-69$} & \multirow{3}{*}{$12 \%$} & \multirow{3}{*}{0.66} & COVID-19 & $3.6 \%$ \\
\hline & & & SARS & $17.6 \%$ \\
\hline & & & MERS & $35 \%$ \\
\hline \multirow{3}{*}{$70-79$} & \multirow{3}{*}{$7 \%$} & \multirow{3}{*}{0.50} & COVID-19 & $8 \%$ \\
\hline & & & SARS & $28 \%$ \\
\hline & & & MERS & $45 \%$ \\
\hline \multirow{3}{*}{ Above 80} & \multirow{3}{*}{$4 \%$} & \multirow{3}{*}{0.42} & COVID-19 & $14.8 \%$ \\
\hline & & & SARS & $26.3 \%$ \\
\hline & & & MERS & $40 \%$ \\
\hline
\end{tabular}


Table 2. Simulation Parameters and Values

\begin{tabular}{|c|c|c|}
\hline Parameter & Description (Source) & Value(s) \\
\hline$R_{0}$ & $\begin{array}{l}\text { Basic reproduction number } \\
\text { (Li et al., 2020; Zhao et al., 2020) }\end{array}$ & 2,4 \\
\hline a & $\begin{array}{l}\text { Incubation rate (per week) } \\
\text { (Yang et al., in press) }\end{array}$ & 1 \\
\hline$\gamma$ & $\begin{array}{l}\text { Recovery rate (per week) } \\
\text { (Yang et al., in press) }\end{array}$ & 1 \\
\hline$I_{0}$ & Initial proportion of infected population & $\begin{array}{c}0.1 \% \\
0.01 \%\end{array}$ \\
\hline$r_{e}$ & Ratio of initially exposed and infected populations & 10 \\
\hline$\left[\beta_{0}, \beta_{\infty}\right]$ & $\begin{array}{l}\text { Initial and final infection rate in the dynamic transmission } \\
\text { model (Kucharski et al., 2020) }\end{array}$ & {$[3,1.5]$} \\
\hline$t_{2}$ & Half-life of decay in the dynamic model (week) & 3,6 \\
\hline$\tau$ & Window length of decay in the dynamic model (week) & $0.5,1$ \\
\hline $\mathrm{N}$ & Population size (million) & 300 \\
\hline$\kappa$ & Weekly subject enrollment in each arm of RCT & 100 \\
\hline$p_{0}^{n v}$ & $\begin{array}{l}\text { Prior probability of having an ineffective nonvaccine anti- } \\
\text { infective therapeutic (Wong et al., 2019) }\end{array}$ & $77 \%$ \\
\hline$p_{0}^{v a c}$ & $\begin{array}{l}\text { Prior probability of having an ineffective vaccine } \\
\text { (Wong et al., 2019) }\end{array}$ & $60 \%$ \\
\hline$\Delta t$ & Time needed to assess the efficacy of the treatment (week) & 1 \\
\hline$\rho$ & $\begin{array}{l}\text { Signal to noise ratio of treatment effect } \\
\text { (Chaudhuri \& Lo, 2018) }\end{array}$ & 0.25 \\
\hline Power $_{\max }$ & $\begin{array}{l}\text { Maximum power of Bayesian decision model } \\
\text { (Isakov et al., 2019) }\end{array}$ & 0.9 \\
\hline$L_{D}$ & Loss per capita from death by infection & 10,100 \\
\hline$L_{I}$ & Loss per capita from being infected & 1 \\
\hline$L_{S}$ & Loss per capita from being susceptible without precaution & 0.2 \\
\hline
\end{tabular}


Table 3. Simulation Results of a Bayesian Adaptive RCT on Nonvaccine Anti-infective Therapeutics Obtained From 10,000 Monte Carlo Runs and assuming $L_{D}=100$

(3)

(4)

(5)

(6)

Epidemic Parameters

\begin{tabular}{|c|c|c|c|c|c|}
\hline$R_{0}$ & $\mu$ & $I_{0}$ & $\begin{array}{c}\text { Sample } \\
\text { Size }\end{array}$ & $\alpha \%$ & Power \% \\
\hline 2 & COVID-19 & $0.1 \%$ & 242 & 7.1 & 90 \\
\hline 4 & COVID-19 & $0.1 \%$ & 158 & 17.3 & 90 \\
\hline$R_{0}(\mathrm{t})$ & COVID-19 & $0.1 \%$ & 176 & 14.4 & 90 \\
\hline
\end{tabular}

(4)

\begin{tabular}{|c|c|c|c|c|c|}
\hline oes & 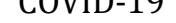 & $0.1 \%$ & & & 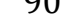 \\
\hline 2 & COVID-19 & $0.01 \%$ & 399 & 1.2 & 90 \\
\hline 4 & COVID-19 & $0.01 \%$ & 274 & 5.0 & 90 \\
\hline$R_{0}(\mathrm{t})$ & COVID-19 & $0.01 \%$ & 304 & 3.6 & 90 \\
\hline
\end{tabular}

\begin{tabular}{|c|c|c|c|c|c|}
\hline & & & & & \\
\hline 2 & SARS & $0.1 \%$ & 164 & 16.3 & 90 \\
\hline 4 & SARS & $0.1 \%$ & 112 & 27.8 & 90 \\
\hline$R_{0}(\mathrm{t})$ & SARS & $0.1 \%$ & 107 & 29.2 & 90 \\
\hline
\end{tabular}

\begin{tabular}{|c|c|c|c|c|c|}
\hline & SARS & 0.170 & & 9.2 & 90 \\
\hline 2 & MERS & $0.1 \%$ & 88 & 35.3 & 90 \\
\hline 4 & MERS & $0.1 \%$ & 63 & 45.2 & 90 \\
\hline$R_{0}(\mathrm{t})$ & MERS & $0.1 \%$ & 44 & 54.3 & 90 \\
\hline
\end{tabular}
(1)
(2)
(3)
(4)
(5) (6)
(7)

\begin{tabular}{|c|c|}
\hline \multicolumn{2}{|c}{ Adaptive } \\
\hline \multicolumn{2}{|c|}{ Sample Size $(\boldsymbol{H}=\mathbf{0})$} \\
\hline $\begin{array}{c}\text { Mean } \\
\text { (SD) }\end{array}$ & $\begin{array}{c}\text { Median } \\
(\mathbf{I Q R})\end{array}$ \\
\hline 135 & 105 \\
$(103)$ & $(63,176)$ \\
\hline 115 & 91 \\
$(83)$ & $(56,149)$ \\
\hline 118 & 95 \\
$(85)$ & $(57,153)$ \\
\hline 150 & 110 \\
$(128)$ & $(64,191)$ \\
\hline 140 & 106 \\
$(110)$ & $(64,180)$ \\
\hline 145 & 108 \\
$(119)$ & $(64,187)$ \\
\hline 117 & 94 \\
$(85)$ & $(57,150)$ \\
\hline 98 & 79 \\
$(72)$ & $(47,128)$ \\
\hline 96 & 78 \\
$(71)$ & $(45,126)$ \\
\hline 87 & 70 \\
$(66)$ & $(40,115)$ \\
\hline 73 & 59 \\
$(59)$ & $(30,100)$ \\
\hline 61 & 48 \\
$(54)$ & $(20,86)$ \\
\hline$(7)$ & $(8)$ \\
\hline & \\
\hline
\end{tabular}

(9)

(43)

000 Monte Carlo Runs) infected subjects. Sample size refers to the number of subjects enrolled in each arm of the RCT. IQR denotes the interquartile range about the median. $R_{0}(t)$ denotes the dynamic transmission model with $t_{2}=3$ weeks, $\tau=1$ week, and $R_{0}(t)$ decreasing from 3 to 1.5 as time $t$ increases. 
Table 4. Simulation Results of Bayesian Adaptive RCT for Vaccines Obtained From 10,000 Monte Carlo Runs and assuming $L_{D}=100$

(1)

\begin{tabular}{|c|c|c|c|c|c|}
\hline \multicolumn{3}{|c|}{ Epidemic Parameters } & \multicolumn{3}{c|}{ Nonadaptive } \\
\cline { 3 - 6 } & $\mu$ & $I_{0}$ & $\begin{array}{c}\text { Sample } \\
\text { Size }\end{array}$ & $\alpha \%$ & Power \% \\
\hline$R_{0}$ & $\mu$ & &
\end{tabular}

\begin{tabular}{|c|c|c|c|c|c|}
\hline 2 & COVID-19 & $0.1 \%$ & 181 & 13.6 & 90 \\
\hline 4 & COVID-19 & $0.1 \%$ & 111 & 28.1 & 90 \\
\hline$R_{0}(\mathrm{t})$ & COVID-19 & $0.1 \%$ & 117 & 26.4 & 90 \\
\hline
\end{tabular}

\begin{tabular}{|c|c|c|c|c|c|}
\hline 4 & COVID-19 & $0.01 \%$ & 232 & 7.9 & 90 \\
\hline$R_{0}(\mathrm{t})$ & COVID-19 & $0.01 \%$ & 244 & 6.9 & 90 \\
\hline
\end{tabular}

\begin{tabular}{|c|c|c|c|c|c|}
\hline 2 & SARS & $0.1 \%$ & 99 & 31.7 & 90 \\
\hline 4 & SARS & $0.1 \%$ & 65 & 44.3 & 90 \\
\hline$R_{0}(\mathrm{t})$ & SARS & $0.1 \%$ & 50 & 51.3 & 90 \\
\hline 2 & MERS & $0.1 \%$ & 27 & 64.2 & 90 \\
\hline 4 & MERS & $0.1 \%$ & 21 & 68.1 & 90 \\
\hline$R_{0}(\mathrm{t})$ & MERS & $0.1 \%$ & 7 & 79.2 & 90 \\
\hline
\end{tabular}

(1) (2) (3) (4) (5)

(6)

Sample Size $(H=0)$

Adaptive (10,000 Monte Carlo Runs)

Note. RCT = randomized clinical trial; $R_{0}$ denotes the basic reproduction number, $\mu$ the disease morality, and $I_{0}$ the proportion of initial infected subjects. Sample size refers to the number of subjects enrolled in each arm of the RCT. IQR denotes the interquartile range about the median. $R_{0}(t)$ denotes the dynamic transmission model with $t_{2}=3$ weeks, $\tau=1$ week, and $R_{0}(\mathrm{t})$ decreasing from 3 to 1.5 as time $t$ increases. 
Table 5. Optimal Sample Size and Type I Error $\alpha$ of Bayesian Nonadaptive RCT for Nonvaccine Antiinfective Therapeutics for Dynamic Transmission Model

\begin{tabular}{|c|c|c|c|c|c|c|c|}
\hline Disease & $I_{0}$ & $\boldsymbol{R}_{\mathbf{0}}$ & $t_{2}$ (week) & $\begin{array}{c}\tau \\
\text { (week) }\end{array}$ & $\begin{array}{c}\text { Sample } \\
\text { Size }\end{array}$ & $\alpha \%$ & $\begin{array}{c}\text { Power } \\
\%\end{array}$ \\
\hline \multirow{6}{*}{$\begin{array}{c}\text { COVID- } \\
19\end{array}$} & \multirow{6}{*}{$0.1 \%$} & 2 & NA & NA & 242 & 7.1 & 90 \\
\hline & & 4 & NA & NA & 158 & 17.3 & 90 \\
\hline & & $R_{0}(\mathrm{t})$ & 3 & 0.5 & 166 & 16.0 & 90 \\
\hline & & $R_{0}(\mathrm{t})$ & 3 & 1 & 176 & 14.4 & 90 \\
\hline & & $R_{0}(\mathrm{t})$ & 6 & 0.5 & 176 & 14.4 & 90 \\
\hline & & $R_{0}(\mathrm{t})$ & 6 & 1 & 177 & 14.2 & 90 \\
\hline \multirow{6}{*}{ SARS } & \multirow{6}{*}{$0.1 \%$} & 2 & NA & NA & 164 & 16.3 & 90 \\
\hline & & 4 & NA & NA & 112 & 27.8 & 90 \\
\hline & & $R_{0}(\mathrm{t})$ & 3 & 0.5 & 100 & 31.3 & 90 \\
\hline & & $R_{0}(\mathrm{t})$ & 3 & 1 & 107 & 29.2 & 90 \\
\hline & & $R_{0}(\mathrm{t})$ & 6 & 0.5 & 118 & 26.2 & 90 \\
\hline & & $R_{0}(\mathrm{t})$ & 6 & 1 & 119 & 25.9 & 90 \\
\hline \multirow{6}{*}{ MERS } & \multirow{6}{*}{$0.1 \%$} & 2 & NA & NA & 88 & 35.3 & 90 \\
\hline & & 4 & NA & NA & 63 & 45.2 & 90 \\
\hline & & $R_{0}(\mathrm{t})$ & 3 & 0.5 & 41 & 55.9 & 90 \\
\hline & & $R_{0}(\mathrm{t})$ & 3 & 1 & 44 & 54.3 & 90 \\
\hline & & $R_{0}(\mathrm{t})$ & 6 & 0.5 & 59 & 47.0 & 90 \\
\hline & & $R_{0}(\mathrm{t})$ & 6 & 1 & 60 & 46.5 & 90 \\
\hline
\end{tabular}

Note. RCT = randomized clinical trial; $\boldsymbol{R}_{0}$ denotes the basic reproduction number, $\mu$ the disease morality, and $I_{0}$ the proportion of initial infected subjects. Sample size refers to the number of subjects enrolled in each arm of the RCT. $R_{0}(t)$ denotes the use of a dynamic transmission model with $\beta_{0}=3, \beta_{\infty}=1.5$. 


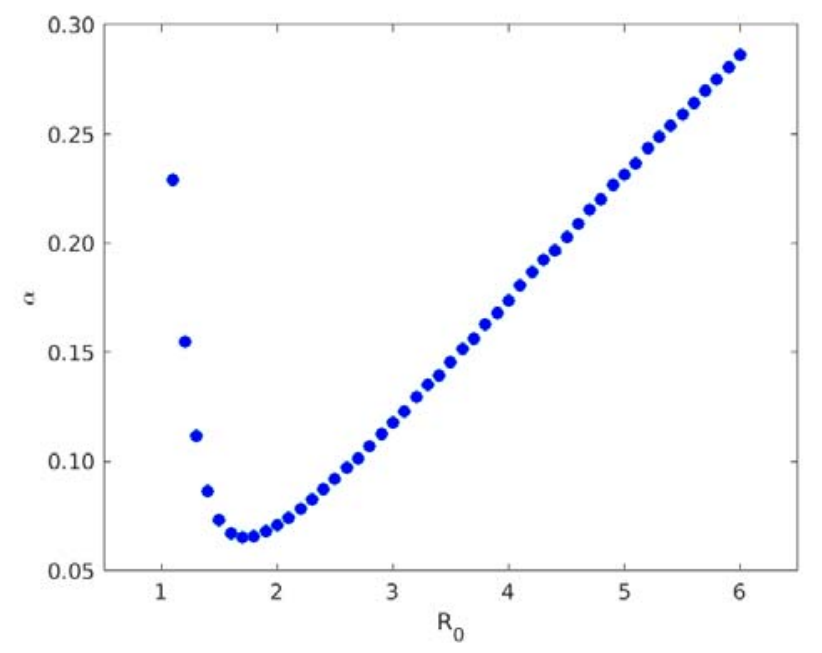

Figure 1. Bayesian Optimal Type I Error Rate. Optimal Type I error rate, $\alpha$, of a nonadaptive Bayesian randomized clinical trial (RCT) vs. basic reproduction number $R_{0}$ (assuming $I_{0}=0.1 \%, L_{D}=100$, disease mortality of COVID-19, and constant $R_{0}$ ). The Bayesian decision model yields a higher $\alpha$ for epidemics with high and low infectivity.

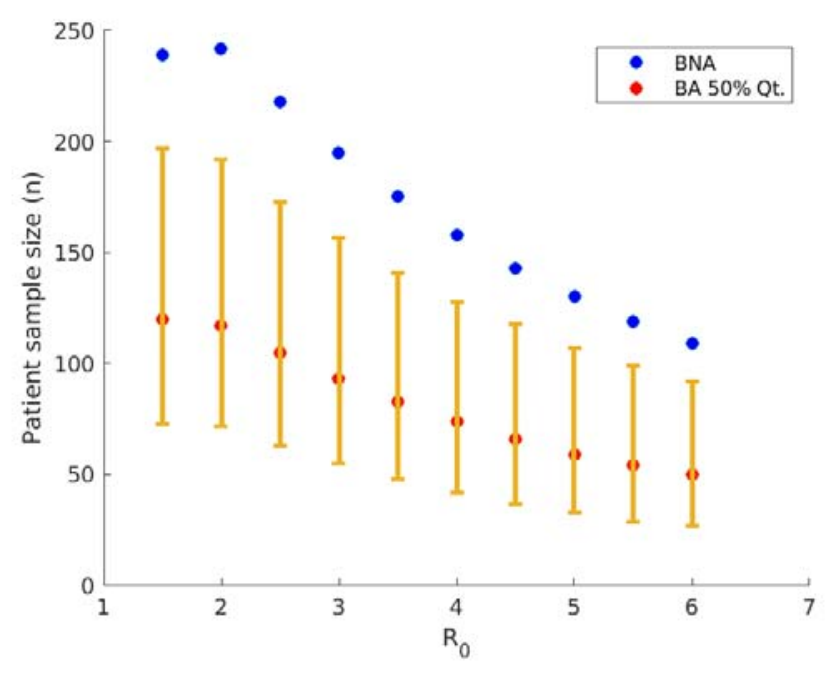

Figure 2. Bayesian Adaptive RCT Patient Sample Size under H = 1. Subject sample size in each arm of a Bayesian adaptive randomized clinical trial (RCT) under $H=1$ decreases with the basic reproduction number $R_{0}$ (assuming $I_{0}=0.1 \%, L_{D}=100$ and disease mortality of COVID-19). BNA denotes Bayesian nonadaptive optimal; BA $50 \%$ denotes median patient size of Bayesian adaptive. The $25 \%$ and $75 \%$ quantiles of Bayesian adaptive patient size are shown as lower and upper ends of the error bar. 


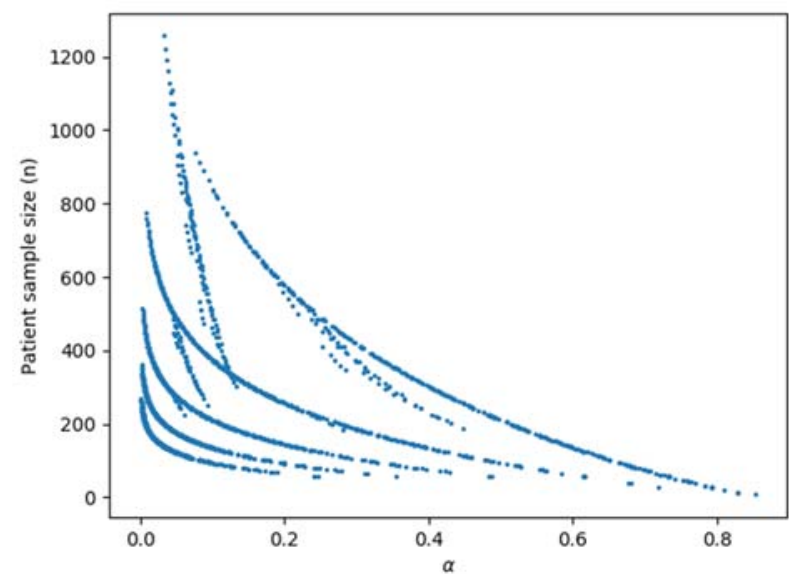

Figure 3. Scatterplot of optimal Type I error $\alpha$ vs. optimal sample size from the five-factor analysis when $R_{0}=2$.
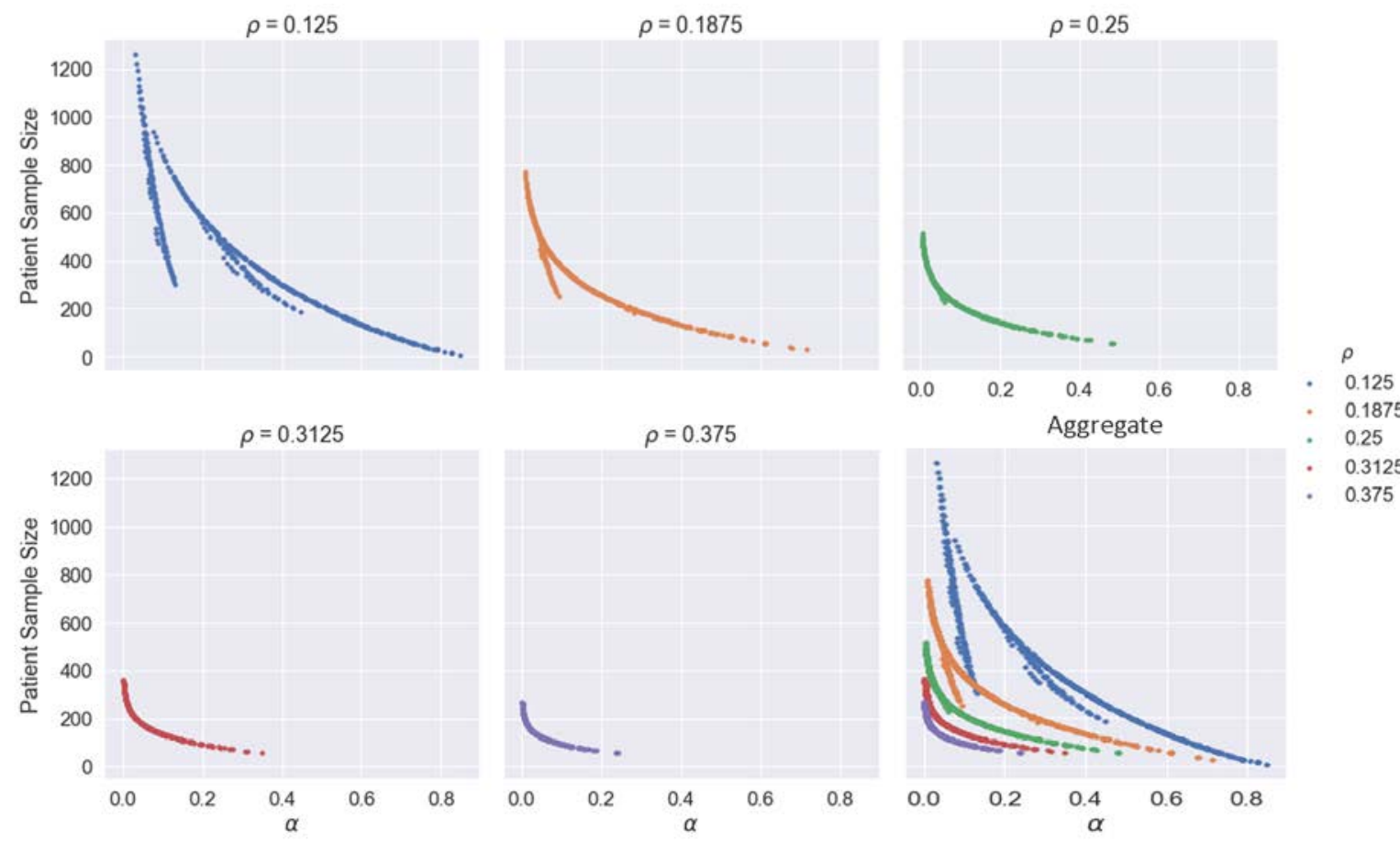

. 0.25

. 0.3125

0.375

Figure 4. Scatterplot of optimal Type I error rate $\alpha$ vs. sample size for different values of $\rho$, signal-tonoise ratio of the treatment effect (Chaudhuri \& Lo, 2018). 

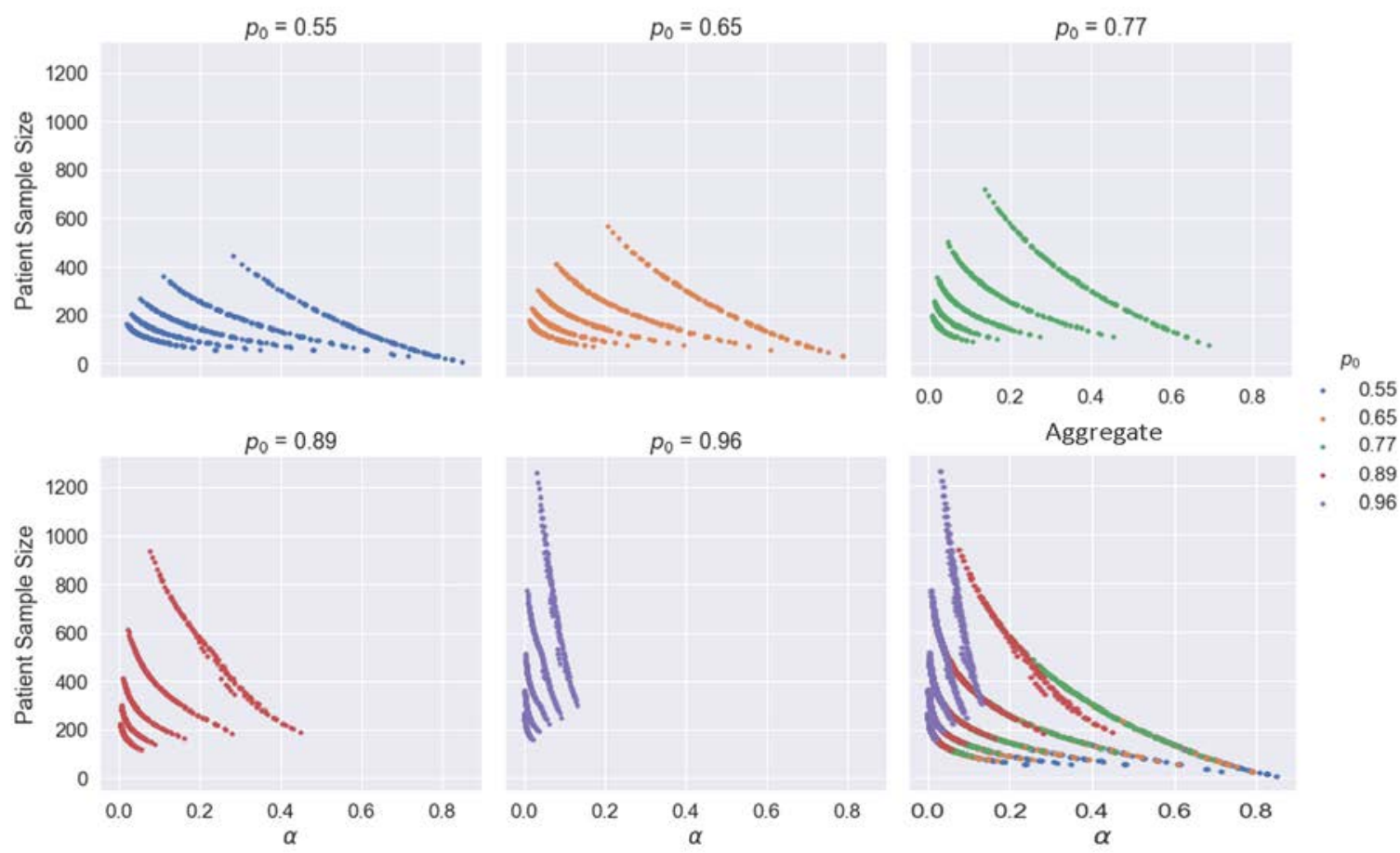

Figure 5. Scatterplot of optimal Type I error rate $\alpha$ vs. sample size for different values of $p_{0}$, Bayesian prior probability of having an ineffective therapeutic.

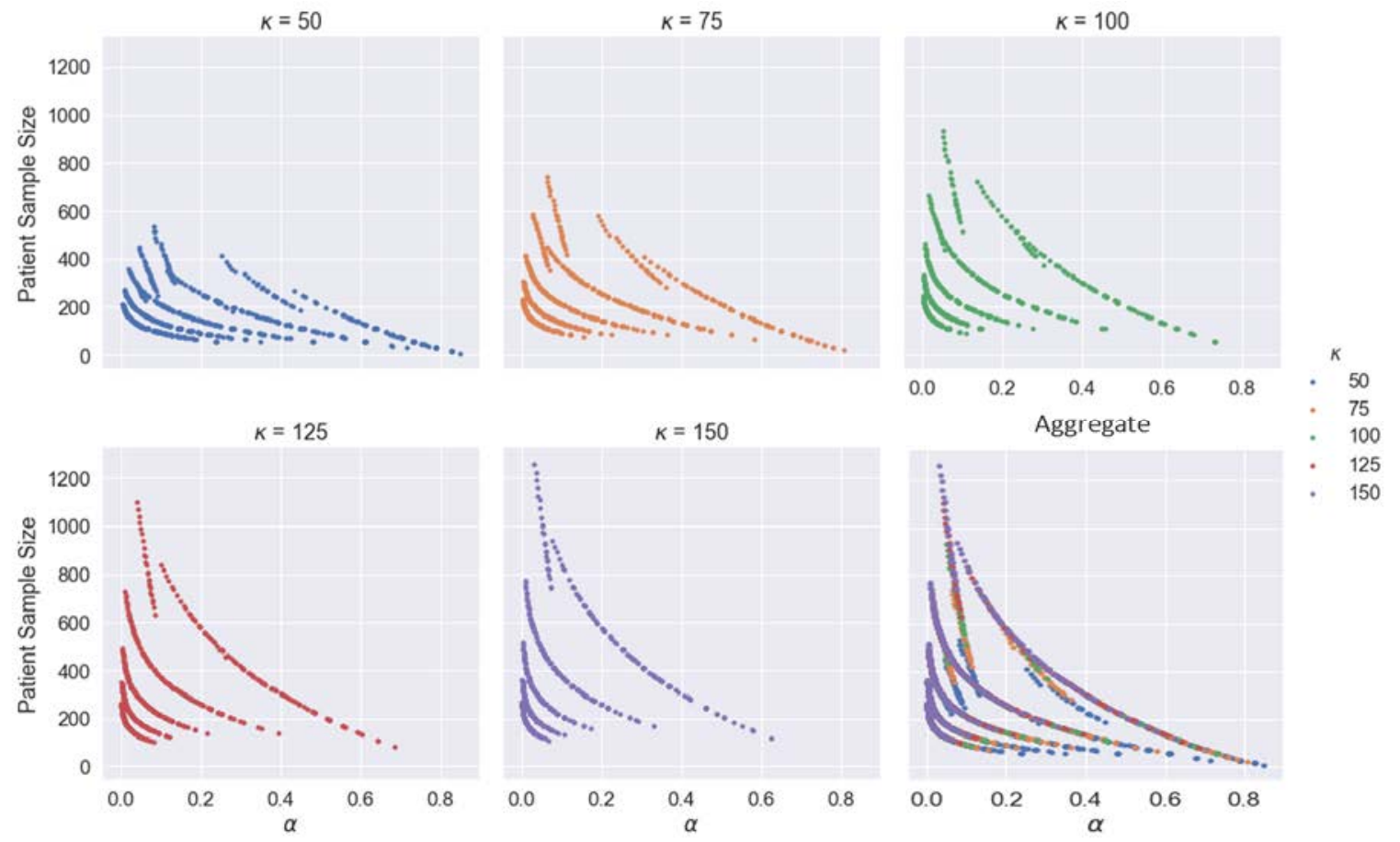

Figure 6. Scatterplot of optimal Type I error rate $\alpha$ vs. sample size for different values of $\kappa$, weekly patient enrollment rate (patients per week) in each arm of a randomized clinical trial. 


\section{Author Disclosure Statements}

S. Chaudhuri, D. Xiao, and Q. Xu report no conflicts.

A. Lo has personal investments in biotechnology companies, biotech venture capital funds, and mutual funds, and is a co-founder and partner of QLS Advisors, a healthcare analytics and consulting company. He is also an advisor to BrightEdge and Thales; an advisor to and investor in BridgeBio Pharma; a director of Roivant Sciences Ltd. and Annual Reviews; chairman emeritus and senior advisor to AlphaSimplex Group; and a member of the Board of Overseers at Beth Israel Deaconess Medical Center. Finally, Lo is a member of the NIH's National Center for Advancing Translational Sciences Advisory Council and Cures Acceleration Network Review Board. During the most recent six-year period, Lo has received speaking/consulting fees, honoraria, or other forms of compensation from: AIG, AlphaSimplex Group, BIS, BridgeBio Capital, Citigroup, Chicago Mercantile Exchange, Financial Times, Harvard University, IMF, National Bank of Belgium, Q Group, Roivant Sciences, Scotia Bank, State Street Bank, University of Chicago, and Yale University. 


\section{References}

$21^{\text {st }}$ Century Cures Act, H.R. 34, 114th Cong. (2016).

Alexander, B. M., Ba, S., Berger, M.S., Berry, D. A., Cavenee, W. K., Chang, S. M., Cloughesy, T. F., Jiang, T., Khasraw, M., Li, W., Mittman, R., Poste, G. H., Wen, P. Y., Yung, W. K. A., Barker, A. D., GBM AGILE Network (2018). Adaptive global innovative learning environment for glioblastoma: GBM AGILE. Clinical Cancer Research, 24(4), 737-743. https://doi.org/10.1158/1078-0432.CCR-17-0764

Barker, A., Sigman, C., Kelloff, G., Hylton, N., Berry, D., \& Esserman, L. (2009). I-SPY 2: An Adaptive breast cancer trial design in the setting of neoadjuvant chemotherapy. Clinical Pharmacology \& Therapeutics, 86(1), 97-100. https://doi.org/10.1038/clpt.2009.68

Berry, D. (2015). The Brave New World of clinical cancer research: Adaptive biomarkerdriven trials integrating clinical practice with clinical research. Molecular Oncology, 9(5), 951-959. https://doi.org/10.1016/j.molonc.2015.02.011

Chaudhuri, S. E., Hauber, B., Mange, B., Zhou, M., Ho, M., Saha, A., Caldwell, B., Benz, H. L., Ruiz, J., Christopher, S., Bardot, D., Sheehan, M., Donnelly, A., McLaughlin, L., Gwinn, K., Sheldon, M., \& A. W. Lo. (2019). Use of Bayesian decision analysis to maximize value in patient-centered randomized clinical trials in Parkinson's Disease, under review.

Chaudhuri, S. E., \& Lo, A. W. (2018). Bayesian adaptive patient-centered clinical trials, under review.

Chaudhuri, S. E., Sheldon, M., Irony, T., Ho, M. and A. W. Lo. (2018). Patient-centered clinical trials. Drug Discovery Today, 23(2), 395-401. https://doi.org/10.1016/j.drudis.2017.09.016 Isakov, L., Lo, A. W., \& Montazerhodjat, V. (2019) Is the FDA too conservative or too aggressive? A Bayesian decision analysis of clinical trial design. Journal of Econometrics, 211(1), 117-136. https://doi.org/10.1016/j.jeconom.2018.12.009

Kucharski, A. J., Russell, T. W., Diamond, C., Liu, Y., Edmunds, J., Funk, S., \& Eggo, R. M. on behalf of the Centre for Mathematical Modelling of Infectious Diseases COVID-19 working group (2020). Early dynamics of transmission and control of COVID-19: A mathematical modelling study. The Lancet Infectious Diseases. Advance online publication. https://doi.org/10.1016/S1473-3099(20)30144-4

Li, Q., ... \& Feng, Z. (2020). Early transmission dynamics in Wuhan, China, of novel coronavirus-infected pneumonia. New England Journal of Medicine, 382, 1199-1207. https://doi.org/10.1056/NEJMoa2001316

Lo, A. (2017). Discussion: New directions for the FDA in the 21st century. Biostatistics, 18(3), 404-407. https://doi.org/10.1093/biostatistics/kxx019

13 May 2020

(C) 2020 by Chaudhuri, Lo, Xiao, and Xu All Rights Reserved

Page 23 of 32 
Montazerhodjat, V., Chaudhuri, S. E., Sargent, D. J., \& Lo, A. W. (2017). Use of Bayesian decision analysis to minimize harm in patient-centered randomized clinical trials in oncology. JAMA Oncology, 3(9), e170123. https://doi.org/10.1001/jamaoncol.2017.0123

Onakpoya, I.J., Heneghan, C.J., \& Aronson, J.K. (2019). Post-marketing withdrawal of 462 medicinal products because of adverse drug reactions: A systematic review of the world literature. BMC Medicine 17(56). https://doi.org/10.1186/s12916-019-1294-9

Onder, G., Rezza, G., \& Brusaferro, S. (2020). Case-fatality rate and characteristics of patients dying in relation to COVID-19 in Italy.JAMA. Advance online publication. https://doi.org/10.1001/jama.2020.4683

Pronker, E. S., Weenen, T. C., Commandeur, H., Claassen, E. H., \& Osterhaus, A. D. (2013) Risk in vaccine research and development quantified. PLoS One, 8(3), e57755. https://doi.org/10.1371/journal.pone.0057755

U.S. Census Bureau. (2018). U.S. Demographics by Age and Sex (Table ID: S0101).Retrieved from https://data.census.gov/

U.S. Food \& Drug Administration. (2013). Structured approach to benefit-risk assessment in drug regulatory decision-making: Draft PDUFA V implementation plan-February 2013, Fiscal Years 2013-2017. http://www.fda.gov/downloads/ForIndustry/UserFees/PrescriptionDrugUserFee/UCM32 9758.pdf.

U.S. Food \& Drug Administration. (2018, January 30). Vaccine product approval process. Retrieved March 8, 2020, from https://www.fda.gov/vaccines-bloodbiologics/development-approval-process-cber/vaccine-product-approval-process

Walker, P. G. T., ..., \& Ghani, A. C. (2020) Report 12: The global impact of COVID-19 and strategies for mitigation and suppression. WHO Collaborating Centre for Infectious Disease Modelling, MRC Centre for Global Infectious Disease Analysis, Abdul Latif Jameel Institute for Disease and Emergency Analytics, Imperial College London. https://www.imperial.ac.uk/mrc-global-infectious-disease-analysis/covid-19/report-12global-impact-covid-19/.

Wong, C. H., Siah, K. W., \& Lo, A. W. (2019). Estimation of clinical trial success rates and $\begin{array}{llll}\text { related } \quad \text { parameters. } & \text { Biostatistics, 20(2), 286. }\end{array}$ https://doi.org/10.1093/biostatistics/kxx069

Wong, C. H., Siah, K. W., \& Lo, A. W. (2020, April 8). Estimating probabilities of success of clinical trials for vaccines and other anti-infective therapeutics. PreprintmedRxiv. https://doi.org/10.1101/2020.04.09.20059600 
World Health Organization. (2020). Coronavirus disease 2019 (COVID-19) situation report59. https://www.who.int/docs/default-source/coronaviruse/situation-reports/20200319sitrep-59-covid-19.pdf?sfvrsn=c3dcdef9 2

World Health Organization. (2003). Consensus document on the epidemiology of severe acute respiratory syndrome (SARS). https://www.who.int/csr/sars/en/WHOconsensus.pdf

World Health Organization. (2019). MERS situation update, December 2019. http://www.emro.who.int/pandemic-epidemic-diseases/mers-cov/mers-situation-updatedecember-2019.html.

Wu, J. T., Leung, K., \& Leung, G. M. (2020). Nowcasting and forecasting the potential domestic and international spread of the 2019-nCoV outbreak originating in Wuhan, China: A modelling study. Lancet, 395(10225), 689-697. https://doi.org/10.1016/S01406736(20)30260-9

Yang, Z., ..., \& He, J. (in press). Modified SEIR and AI prediction of the epidemics trend of COVID-19 in China under public health interventions. Journal of Thoracic Disease. http://dx.doi.org/10.21037/jtd.2020.02.64

Zhao, S., Lin, Q., Ran, J., Musa, S. S., Yang, G., Wang, W., Lou, Y., Gao, D., Yang, L., He, D., \& Wang, M. H. (2020). Preliminary estimation of the basic reproduction number of novel coronavirus (2019-nCoV) in China, from 2019 to 2020: A data-driven analysis in the early phase of the outbreak. International Journal of Infectious Diseases, 92, 214-217. https://doi.org/10.1016/j.ijid.2020.01.050 


\section{Appendix}

Table S1. Baseline and Alternative Parameter Values Used in the Five-Factor Analysis

\begin{tabular}{|c|l|c|l|}
\hline Parameter & \multicolumn{1}{|c|}{ Description } & \multicolumn{1}{|c|}{$\begin{array}{c}\text { Baseline } \\
\text { Value }\end{array}$} & \multicolumn{1}{|c|}{$\begin{array}{c}\text { Alternative } \\
\text { Values }\end{array}$} \\
\hline a & $\begin{array}{l}\text { Incubation rate (per week) (Yang et } \\
\text { al. 2020) }\end{array}$ & 1 & $\begin{array}{l}0.5, \quad 0.75, \\
1.25,1.5\end{array}$ \\
\hline$\kappa$ & $\begin{array}{l}\text { Weekly subject enrollment in each arm } \\
\text { of RCT (per week) }\end{array}$ & 100 & $\begin{array}{l}50,75, \quad 125, \\
150\end{array}$ \\
\hline$p_{0}^{n v}$ & $\begin{array}{l}\text { Prior probability of having an ineffective } \\
\text { nonvaccine anti-infective therapy (Wong } \\
\text { et al., 2019) }\end{array}$ & $77 \%$ & $\begin{array}{l}54.90 \%, \\
65.45 \%, \\
88.55 \%, \\
96.25 \%\end{array}$ \\
\hline$\rho$ & $\begin{array}{l}\text { Signal-to-noise ratio of treatment effect } \\
\text { (Chaudhuri \& Lo, 2018) }\end{array}$ & 0.25 & $\begin{array}{l}0.125, \\
0.1875, \\
0.3125,0.375\end{array}$ \\
\hline \multirow{2}{*}{$\Delta t$} & $\begin{array}{l}\text { Time needed to assess the efficacy of the } \\
\text { treatment (week) }\end{array}$ & 1 & $0,0.5,1.5,2$ \\
\hline
\end{tabular}


Table S2. Optimal Sample Size and Type I error rate $\alpha$ for Bayesian Nonadaptive RCT on Anti-infective Therapeutics With $R_{0}$ (Basic Reproduction Number) Close to 1

\begin{tabular}{|c|c|c|c|c|c|}
\hline Disease & $R_{0}$ & $I_{0}$ & Sample Size & $\alpha \%$ & Power \% \\
\hline \multirow{10}{*}{ COVID-19 } & 1.25 & $0.1 \%$ & 185 & 13.1 & 90 \\
\hline & 1.5 & $0.1 \%$ & 239 & 7.3 & 90 \\
\hline & 1.75 & $0.1 \%$ & 250 & 6.5 & 90 \\
\hline & 2 & $0.1 \%$ & 242 & 7.1 & 90 \\
\hline & 4 & $0.1 \%$ & 158 & 17.3 & 90 \\
\hline & 1.25 & $0.01 \%$ & 233 & 7.8 & 90 \\
\hline & 1.5 & $0.01 \%$ & 340 & 2.4 & 90 \\
\hline & 1.75 & $0.01 \%$ & 395 & 1.3 & 90 \\
\hline & 2 & $0.01 \%$ & 399 & 1.2 & 90 \\
\hline & 4 & $0.01 \%$ & 274 & 5.0 & 90 \\
\hline \multirow{5}{*}{ SARS } & 1.25 & $0.1 \%$ & 69 & 42.6 & 90 \\
\hline & 1.5 & $0.1 \%$ & 140 & 20.9 & 90 \\
\hline & 1.75 & $0.1 \%$ & 162 & 16.6 & 90 \\
\hline & 2 & $0.1 \%$ & 164 & 16.3 & 90 \\
\hline & 4 & $0.1 \%$ & 112 & 27.8 & 90 \\
\hline \multirow{5}{*}{ MERS } & 1.25 & $0.1 \%$ & 6 & 80.2 & 90 \\
\hline & 1.5 & $0.1 \%$ & 51 & 50.8 & 90 \\
\hline & 1.75 & $0.1 \%$ & 80 & 38.2 & 90 \\
\hline & 2 & $0.1 \%$ & 88 & 35.3 & 90 \\
\hline & 4 & $0.1 \%$ & 63 & 45.2 & 90 \\
\hline
\end{tabular}

Note. RCT $=$ randomized clinical trial; $\mu$ denotes the disease morality and $I_{0}$ the proportion of initial infected subjects. Sample size denotes the number of subjects enrolled in each arm of the RCT. 
Table S3. Simulation Results of Bayesian adaptive RCT on Nonvaccine Anti-infective Therapeutics Obtained From 10,000 Monte Carlo Runs and assuming $L_{D}=10$

\begin{tabular}{|c|c|c|c|c|c|c|c|c|c|c|c|}
\hline \multirow{2}{*}{\multicolumn{3}{|c|}{ Epidemic Parameters }} & \multirow{2}{*}{\multicolumn{3}{|c|}{ Nonadaptive }} & \multicolumn{6}{|c|}{ Adaptive (10,000 Monte Carlo Runs) } \\
\hline & & & & & & \multicolumn{2}{|c|}{ Sample Size $(H=0)$} & \multicolumn{2}{|c|}{ Sample Size $(H=1)$} & \multirow[b]{2}{*}{$\alpha \%$} & \multirow[b]{2}{*}{ Power \% } \\
\hline$R_{0}$ & $\mu$ & $I_{0}$ & $\begin{array}{c}\text { Sample } \\
\text { Size } \\
\end{array}$ & $\alpha \%$ & Power \% & $\begin{array}{l}\text { Mean } \\
(S D)\end{array}$ & $\begin{array}{c}\text { Median } \\
\text { (IQR) }\end{array}$ & $\begin{array}{l}\text { Mean } \\
(S D)\end{array}$ & $\begin{array}{l}\text { Median } \\
\text { (IQR) }\end{array}$ & & \\
\hline 2 & COVID-19 & $0.1 \%$ & 281 & 4.7 & 90 & $\begin{array}{c}141 \\
(113)\end{array}$ & $\begin{array}{c}107 \\
(63,182) \\
\end{array}$ & $\begin{array}{c}175 \\
(121)\end{array}$ & $\begin{array}{c}142 \\
(90,226)\end{array}$ & 3.7 & 91.3 \\
\hline 4 & COVID-19 & $0.1 \%$ & 176 & 14.4 & 90 & $\begin{array}{l}119 \\
(86)\end{array}$ & $\begin{array}{c}96 \\
(59,153)\end{array}$ & $\begin{array}{l}108 \\
(85)\end{array}$ & $\begin{array}{c}83 \\
(48,139)\end{array}$ & 11.9 & 92.3 \\
\hline$R_{0}(\mathrm{t})$ & COVID-19 & $0.1 \%$ & 213 & 9.7 & 90 & $\begin{array}{l}129 \\
(95)\end{array}$ & $\begin{array}{c}101 \\
(61,168)\end{array}$ & $\begin{array}{l}131 \\
(97)\end{array}$ & $\begin{array}{c}103 \\
(63,168)\end{array}$ & 8.3 & 91.7 \\
\hline 2 & COVID-19 & $0.01 \%$ & 433 & 0.8 & 90 & $\begin{array}{c}150 \\
(128)\end{array}$ & $\begin{array}{c}109 \\
(64,191)\end{array}$ & $\begin{array}{c}272 \\
(166)\end{array}$ & $\begin{array}{c}223 \\
(156,348)\end{array}$ & 0.6 & 91.1 \\
\hline 4 & COVID-19 & $0.01 \%$ & 290 & 4.2 & 90 & $\begin{array}{c}143 \\
(113)\end{array}$ & $\begin{array}{c}108 \\
(63,185) \\
\end{array}$ & $\begin{array}{c}177 \\
(119)\end{array}$ & $\begin{array}{c}145 \\
(92,229) \\
\end{array}$ & 3.3 & 91.1 \\
\hline$R_{0}(\mathrm{t})$ & COVID-19 & $0.01 \%$ & 345 & 2.3 & 90 & $\begin{array}{c}146 \\
(118)\end{array}$ & $\begin{array}{c}111 \\
(65,188)\end{array}$ & $\begin{array}{c}217 \\
(119)\end{array}$ & $\begin{array}{c}181 \\
(117,282)\end{array}$ & 1.9 & 91.0 \\
\hline 2 & SARS & $0.1 \%$ & 262 & 5.7 & 90 & $\begin{array}{c}138 \\
(107)\end{array}$ & $\begin{array}{c}107 \\
(63,179)\end{array}$ & $\begin{array}{c}161 \\
(115)\end{array}$ & $\begin{array}{c}130 \\
(81,207)\end{array}$ & 4.6 & 91.4 \\
\hline 4 & SARS & $0.1 \%$ & 167 & 15.8 & 90 & $\begin{array}{l}118 \\
(86)\end{array}$ & $\begin{array}{c}94 \\
(57,154)\end{array}$ & $\begin{array}{l}102 \\
(82)\end{array}$ & $\begin{array}{c}77 \\
(46,133)\end{array}$ & 13.6 & 92.3 \\
\hline$R_{0}(\mathrm{t})$ & SARS & $0.1 \%$ & 194 & 11.9 & 90 & $\begin{array}{l}126 \\
(93)\end{array}$ & $\begin{array}{c}100 \\
(60,165)\end{array}$ & $\begin{array}{l}118 \\
(90)\end{array}$ & $\begin{array}{c}92 \\
(55,154)\end{array}$ & 9.7 & 92.5 \\
\hline 2 & MERS & $0.1 \%$ & 227 & 8.4 & 90 & $\begin{array}{l}130 \\
(97)\end{array}$ & $\begin{array}{c}102 \\
(62,167)\end{array}$ & $\begin{array}{c}140 \\
(101)\end{array}$ & $\begin{array}{c}112 \\
(68,181)\end{array}$ & 7.4 & 91.6 \\
\hline 4 & MERS & $0.1 \%$ & 149 & 19.0 & 90 & $\begin{array}{l}110 \\
(78)\end{array}$ & $\begin{array}{c}89 \\
(55,142) \\
\end{array}$ & $\begin{array}{c}93 \\
(78)\end{array}$ & $\begin{array}{c}69 \\
(39,122) \\
\end{array}$ & 16.1 & 92.7 \\
\hline$R_{0}(\mathrm{t})$ & MERS & $0.1 \%$ & 163 & 16.5 & 90 & $\begin{array}{l}116 \\
(84)\end{array}$ & $\begin{array}{c}93 \\
(56,151)\end{array}$ & $\begin{array}{l}101 \\
(82)\end{array}$ & $\begin{array}{c}78 \\
(45,121)\end{array}$ & 13.7 & 92.2 \\
\hline
\end{tabular}

(1)

(2)

(3)

(4)

(5)

(6)

(7)

(8)

(9)

$(10)$

(11)

(12)

Note. RCT $=$ randomized clinical trial; $R_{0}$ denotes the basic reproduction number, $\mu$ the disease morality, and $I_{0}$ the proportion of initia infected subjects. Sample size refers to the number of subjects enrolled in each arm of the RCT. IQR denotes the interquartile range about the median. $R_{0}(\mathrm{t})$ denotes the dynamic transmission model with $t_{2}=3$ weeks, $\tau=1$ week, and $R_{0}(\mathrm{t})$ decreasing from 3 to 1.5 as time $t$ increases. 
Table S4. Simulation Results of Bayesian Adaptive RCT on Vaccines Obtained From 10,000 Monte Carlo Runs and assuming $L_{D}=10$

\begin{tabular}{|c|c|c|c|c|c|c|c|c|c|c|c|}
\hline \multirow{2}{*}{\multicolumn{3}{|c|}{ Epidemic Parameters }} & \multirow{2}{*}{\multicolumn{3}{|c|}{ Nonadaptive }} & \multicolumn{6}{|c|}{ Adaptive (10,000 Monte Carlo Runs) } \\
\hline & & & & & & \multicolumn{2}{|c|}{ Sample Size $(H=0)$} & \multicolumn{2}{|c|}{ Sample Size $(H=1)$} & \multirow[b]{2}{*}{$\alpha \%$} & \multirow[b]{2}{*}{ Power \% } \\
\hline$R_{0}$ & $\mu$ & $I_{0}$ & $\begin{array}{c}\text { Sample } \\
\text { Size }\end{array}$ & $\alpha \%$ & Power \% & $\begin{array}{c}\text { Mean } \\
(S D)\end{array}$ & $\begin{array}{c}\text { Median } \\
\text { (IQR) }\end{array}$ & $\begin{array}{l}\text { Mean } \\
(S D)\end{array}$ & $\begin{array}{c}\text { Median } \\
\text { (IQR) }\end{array}$ & & \\
\hline 2 & COVID-19 & $0.1 \%$ & 221 & 8.9 & 90 & $\begin{array}{l}130 \\
(98)\end{array}$ & $\begin{array}{c}103 \\
(62,167)\end{array}$ & $\begin{array}{c}136 \\
(100)\end{array}$ & $\begin{array}{c}109 \\
(66,176)\end{array}$ & 7.5 & 91.9 \\
\hline 4 & COVID-19 & $0.1 \%$ & 129 & 23.4 & 90 & $\begin{array}{l}105 \\
(78)\end{array}$ & $\begin{array}{c}84 \\
(51,135)\end{array}$ & $\begin{array}{c}80 \\
(70)\end{array}$ & $\begin{array}{c}58 \\
(33,105) \\
\end{array}$ & 19.2 & 92.2 \\
\hline$R_{0}(\mathrm{t})$ & COVID-19 & $0.1 \%$ & 151 & 18.7 & 90 & $\begin{array}{l}114 \\
(83)\end{array}$ & $\begin{array}{c}92 \\
(56,149)\end{array}$ & $\begin{array}{c}94 \\
(78) \\
\end{array}$ & $\begin{array}{c}69 \\
(40,123) \\
\end{array}$ & 15.4 & 92.4 \\
\hline 2 & COVID-19 & $0.01 \%$ & 377 & 1.6 & 90 & $\begin{array}{c}148 \\
(126)\end{array}$ & $\begin{array}{c}110 \\
(64,187)\end{array}$ & $\begin{array}{c}236 \\
(150)\end{array}$ & $\begin{array}{c}200 \\
(130,300)\end{array}$ & 1.2 & 90.8 \\
\hline 4 & COVID-19 & $0.01 \%$ & 247 & 6.7 & 90 & $\begin{array}{c}135 \\
(105)\end{array}$ & $\begin{array}{c}103 \\
(63,175)\end{array}$ & $\begin{array}{c}150 \\
(106)\end{array}$ & $\begin{array}{c}121 \\
(75,196) \\
\end{array}$ & 5.4 & 91.3 \\
\hline$R_{0}(\mathrm{t})$ & COVID-19 & $0.01 \%$ & 281 & 4.6 & 90 & $\begin{array}{c}139 \\
(109)\end{array}$ & $\begin{array}{c}107 \\
(62,181)\end{array}$ & $\begin{array}{c}170 \\
(116)\end{array}$ & $\begin{array}{c}139 \\
(88,220)\end{array}$ & 3.7 & 91.4 \\
\hline 2 & SARS & $0.1 \%$ & 201 & 11.0 & 90 & $\begin{array}{l}127 \\
(94)\end{array}$ & $\begin{array}{c}100 \\
(61,165)\end{array}$ & $\begin{array}{l}122 \\
(91) \\
\end{array}$ & $\begin{array}{c}96 \\
(58,159)\end{array}$ & 9.9 & 91.9 \\
\hline 4 & SARS & $0.1 \%$ & 120 & 25.6 & 90 & $\begin{array}{l}101 \\
(74)\end{array}$ & $\begin{array}{c}81 \\
(49,131)\end{array}$ & $\begin{array}{c}76 \\
(68)\end{array}$ & $\begin{array}{c}55 \\
(30,100) \\
\end{array}$ & 20.9 & 93.4 \\
\hline$R_{0}(\mathrm{t})$ & SARS & $0.1 \%$ & 134 & 22.2 & 90 & $\begin{array}{l}106 \\
(76)\end{array}$ & $\begin{array}{c}86 \\
(52,139)\end{array}$ & $\begin{array}{c}83 \\
(72)\end{array}$ & $\begin{array}{c}60 \\
(33,108)\end{array}$ & 18.3 & 92.5 \\
\hline 2 & MERS & $0.1 \%$ & 166 & 16.0 & 90 & $\begin{array}{l}116 \\
(84)\end{array}$ & $\begin{array}{c}92 \\
(56,152)\end{array}$ & $\begin{array}{l}102 \\
(84)\end{array}$ & $\begin{array}{c}77 \\
(45,132)\end{array}$ & 13.5 & 92.2 \\
\hline 4 & MERS & $0.1 \%$ & 103 & 30.4 & 90 & $\begin{array}{c}93 \\
(70)\end{array}$ & $\begin{array}{c}75 \\
(44,122) \\
\end{array}$ & $\begin{array}{c}67 \\
(63) \\
\end{array}$ & $\begin{array}{c}46 \\
(24,88)\end{array}$ & 25.7 & 93.5 \\
\hline$R_{0}(\mathrm{t})$ & MERS & $0.1 \%$ & 105 & 29.8 & 90 & $\begin{array}{c}96 \\
(71)\end{array}$ & $\begin{array}{c}77 \\
(45,125) \\
\end{array}$ & $\begin{array}{c}68 \\
(62) \\
\end{array}$ & $\begin{array}{c}48 \\
(25,91) \\
\end{array}$ & 25.4 & 93.3 \\
\hline
\end{tabular}

(1) (2) $\quad(2)$

(3) (3)

(4)

(5)

(6)

(7)

(8)

(9)

$(10)$

(11)

Note. RCT $=$ randomized clinical trial; $R_{0}$ denotes the basic reproduction number, $\mu$ the disease morality, and $I_{0}$ the proportion of initial infected subjects. Sample size refers to the number of subjects enrolled in each arm of the RCT. IQR denotes the interquartile range about the median. $R_{0}(\mathrm{t})$ denotes the dynamic transmission model with $t_{2}=3$ weeks, $\tau=1$ week, and $R_{0}(\mathrm{t})$ decreasing from 3 to 1.5 as time $t$ increases. 
Table S5. Summary statistics of optimal Type I error rate (top) and sample size (bottom)

\begin{tabular}{|l|l|l|l|l|l|l|l|}
\hline Min & $\mathbf{1 0} \%$ & $\mathbf{2 5 \%}$ & $\mathbf{5 0 \%}$ & $\mathbf{7 5 \%}$ & $\mathbf{9 0 \%}$ & Max & Baseline \\
\hline $0.1 \%$ & $1.0 \%$ & $2.7 \%$ & $7.1 \%$ & $19.9 \%$ & $40.8 \%$ & $85.3 \%$ & $7.1 \%$ \\
\hline
\end{tabular}

\begin{tabular}{|l|l|l|l|l|l|l|l|}
\hline Min & $\mathbf{1 0} \%$ & $\mathbf{2 5 \%}$ & $\mathbf{5 0 \%}$ & $\mathbf{7 5 \%}$ & $\mathbf{9 0 \%}$ & Max & Baseline \\
\hline 7 & 112 & 153 & 220 & 329 & 486 & 1,257 & 242 \\
\hline
\end{tabular}


Figure S1. Scatterplot of optimal Type I error rate $\alpha$ vs. sample size for different values of $\Delta t$, the time needed to assess the treatment efficacy (week).
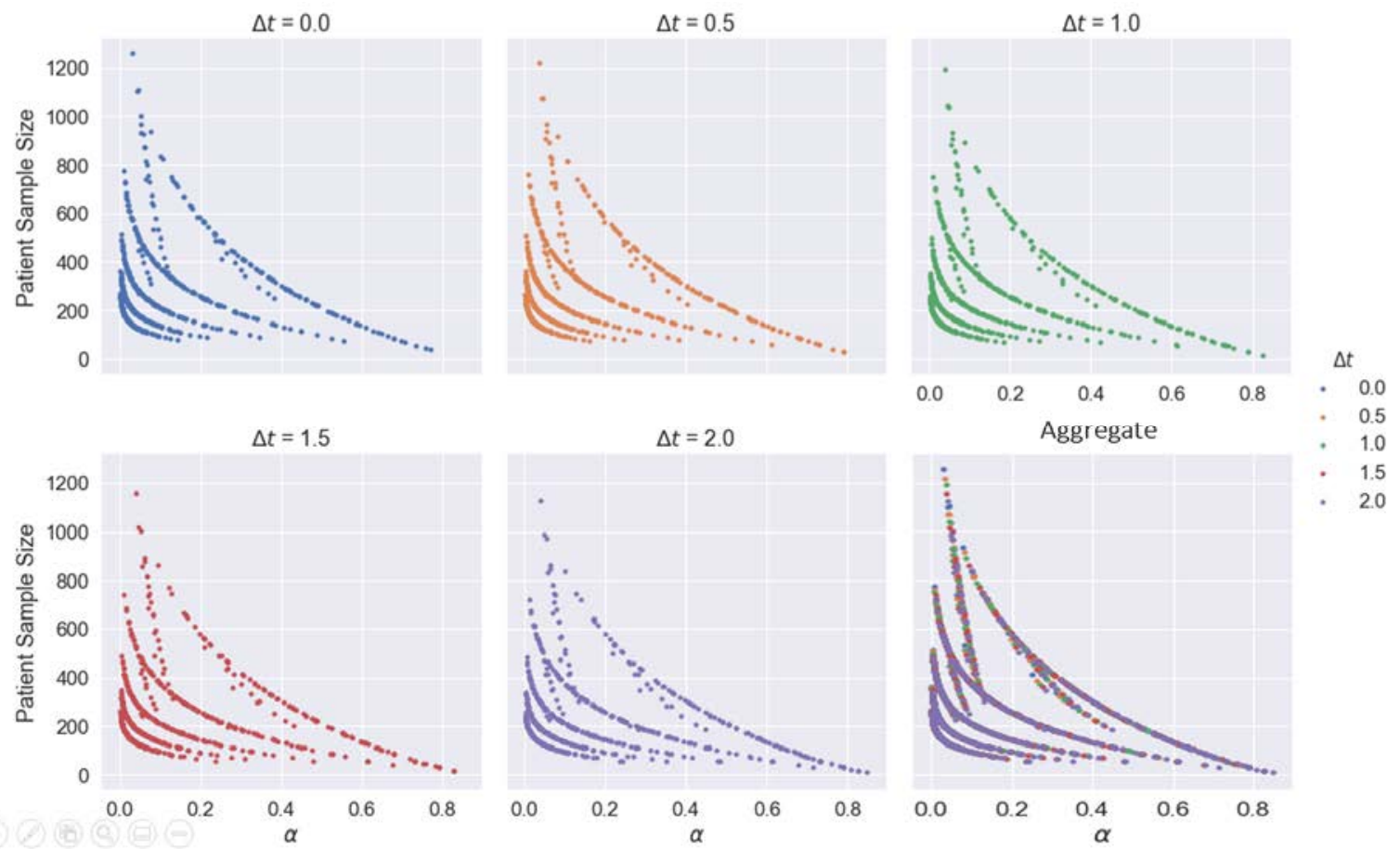

Figure S2. Scatterplot of optimal Type I error rate $\alpha$ vs. sample size for different values of $a$, the incubation period (week) of the disease.
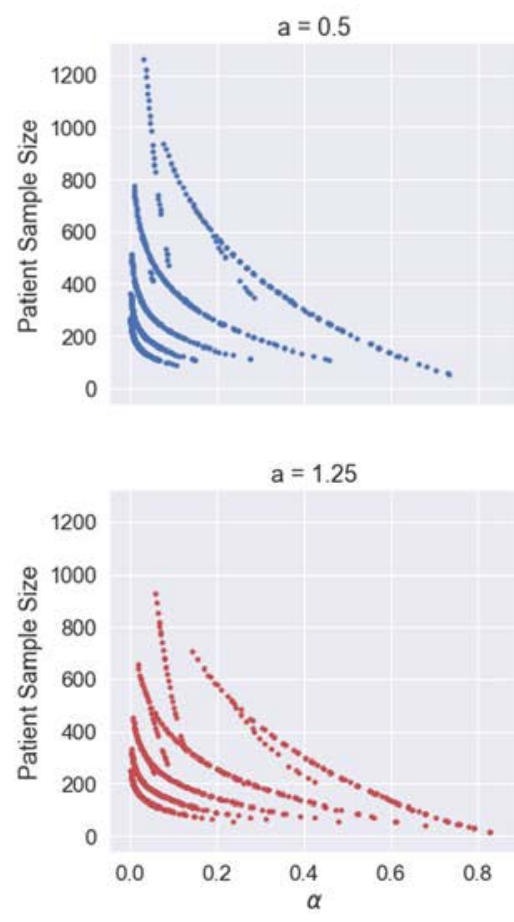
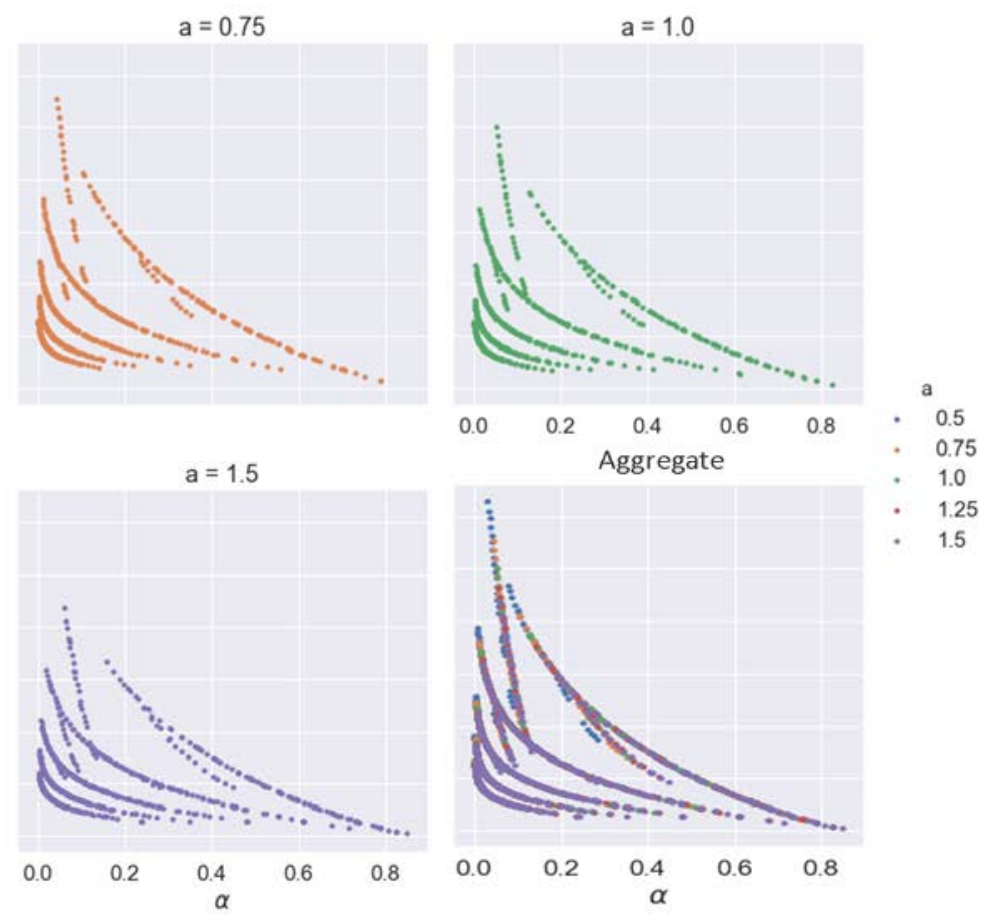
Figure S3. Optimal Type I Error vs. $R_{0}$ for Absolute Risk of Being Susceptible. Optimal Type I error rate $\alpha$ of nonadaptive Bayesian randomized clinical trial monotonically increases with the basic reproduction number $R_{0}$ (assuming $I_{0}=0.1 \%, L_{D}=100$ and disease mortality of COVID-19) if we define the loss of making a Type I error as the absolute risk of being susceptible $S(t) N L_{S}$. This alternative definition is not very realistic. For an epidemic with $R_{0}<2$, the loss of Type I error converges to a large positive value $S(T) N L_{S}$ as time approaches the end of the epidemic outbreak. However, at the end of the outbreak, there are no more infected patients and thus no susceptible subjects. Therefore, the loss of Type I error should approach zero as $t \rightarrow T$. This is the case for the excess risk of susceptibility $(S(t)-S(T)) N L_{S}$ but not for the absolute risk of susceptibility $S(t) N L_{S}$.

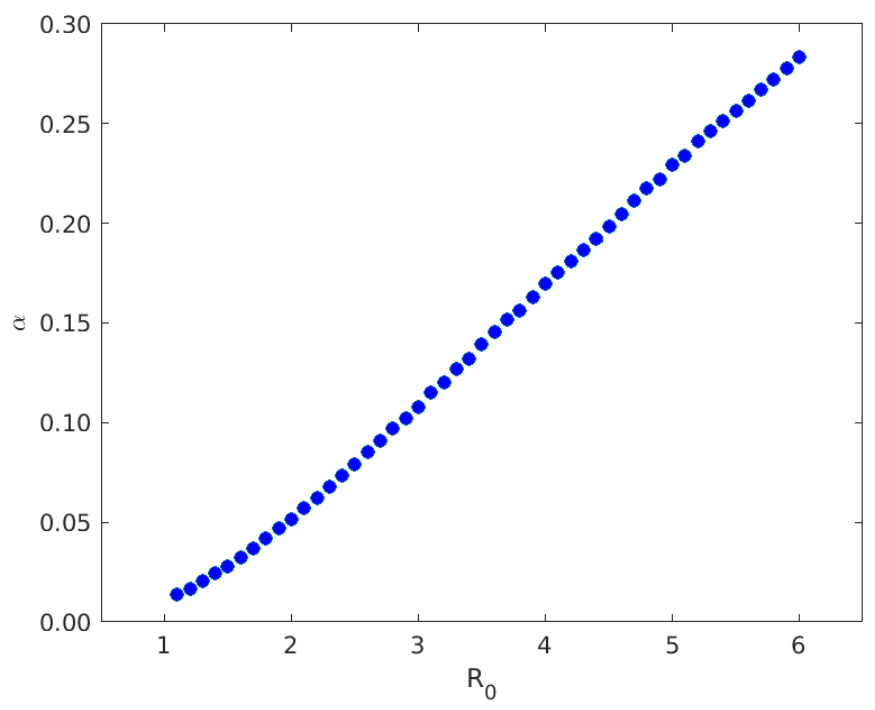

